\title{
Three-dimensional vortex analysis and aeroacoustic source characterization of jet core breakdown
}

\author{
Daniele Violato and Fulvio Scarano \\ Department of Aerospace Engineering, Delft University of Technology, Kluyverweg 2, \\ 2629 HS, Delft, The Netherlands
}

(Received 1 June 2012; accepted 28 November 2012; published online 22 January 2013)

\begin{abstract}
The three-dimensional behavior of jet core breakdown is investigated with experiments conducted on a free water jet at $R e=5000$ by time-resolved tomographic particle image velocimetry (TR-TOMO PIV). The investigated domain encompasses the range between 0 and 10 jet diameters. The characteristic pulsatile motion of vortex ring shedding and pairing culminates with the growth of four primary inplane and out-of-plane azimuthal waves and leads to the formation of streamwise vortices. Vortex ring humps are tilted and ejected along the axial direction as they are subjected to higher axial velocities. By the end of the potential core, this process causes the breakdown of the vortex ring regime and the onset of streamwise filaments oriented at $30^{\circ}-45^{\circ}$ to the jet axis and " $\mathrm{C}$ " shaped peripheral structures. The latter re-organize further downstream in filaments oriented along the azimuthal direction at the jet periphery. Instead, in the vicinity of the jet axis the filaments do not exhibit any preferential direction resembling the isotropic turbulent regime. Following Powell's aeroacoustic analogy, the instantaneous spatial distribution of the acoustic source term is mapped by the second time derivative of the Lamb vector, revealing the highest activity during vortex ring breakdown. A three-dimensional modal analysis of velocity, vorticity, Lamb vector, and Lamb vector second time derivative fields is conducted by proper orthogonal decomposition (POD) within the first 10 modes. The decomposed velocity fluctuations describe a helical organization in the region of the jet core-breakdown and, further downstream, jet axis flapping and precession motions. By the end of the potential core, vorticity modes show that vortex rings are dominated by travelling waves of radial and axial vorticity with a characteristic $40^{\circ}-45^{\circ}$ inclination to the jet axis. The Lamb vector and the Lamb vector second time derivative modes exhibit similar patterns for the azimuthal component, whereas the vortex ring coherence is described by the radial and the axial components. While velocity, vorticity, and Lamb vector modes are typically associated with Strouhal numbers $(S t)$ smaller than 0.9 , the modes of the Lamb vector second time derivative are also related to higher frequencies $(1.05 \leq S t \leq 1.9)$ ascribed to the three-dimensional travelling waves. Far-field acoustic predictions are obtained on the basis of direct evaluation of Powell's analogy with TR-TOMO PIV data. The spectral analysis returns peaks at pairing $(S t=0.36)$ and shedding $(S t=0.72)$ frequency. A broader distribution with a hump between $S t=1$ and 2.25 is observed, which corresponds to the breakdown of ring vortices. @ 2013 American Institute of Physics. [http://dx.doi.org/10.1063/1.4773444]
\end{abstract}

\section{INTRODUCTION}

Acoustic emissions in subsonic jet flows are believed to be related to the dynamical behavior of large-scale flow structures and their interactions. Intense sound radiation originates with the collapse of the annular mixing-layer at the end of the potential core (Juve et al. ${ }^{1}$ Hussain and Zaman, ${ }^{2}$ Hussain, ${ }^{3}$ Bogey et al., ${ }^{4}$ and Jordan et al. ${ }^{5}$ ). Experimental studies describing the large-scale patterns 
and dynamical events across the end of the potential core are thus relevant to understand the physical mechanism associated with sound production.

In the region downstream the shedding and pairing of axisymmetric vortices, Hussain and Zaman $^{2}$ identified toroidal structures developing azimuthal lobes, which split into coherent substructures by the end of the potential core. The same authors argued that the formation of these lobes and the breakdown of the vortices are more important in the production of aerodynamic noise than vortex pairing. With hydrogen bubble visualizations, Yule ${ }^{6}$ found that the end of the potential core is characterized by the loss of circumferential coherence (Liepmann and Gharib ${ }^{7}$ ) and the formation of smaller scales that ejects bursts of fluids. Juve et al. ${ }^{1}$ observed that sudden decelerations occur near the end of the potential core, which are due to the engulfment of fluid by toroidal structures with an intermittent noise emission signature. Using planar particle image velocimetry (PIV) and flow visualization, Liepmann and Gharib ${ }^{7}$ described how the streamwise vorticity relates to the entrainment process beyond the end of the potential core. Kyle and Shreenivasan ${ }^{8}$ visualized an energetic and highly regular pairing process that leads to the early and abrupt breakdown of the potential core. With aeroacoustic predictions based on large eddy simulation, Bogey et al. ${ }^{4}$ conjectured that the acoustic generation may involve the sudden acceleration and stretching of vortical structures when coming towards the jet axial region at the end of the potential core.

Further experiments focused on the region downstream of the axisymmetric regime where specific strategies were adopted to reconstruct three-dimensional flow features from point-wise planar measurements. Matsuda and Sakakibara ${ }^{9}$ produced a 3D representation of the turbulent coherent structures combining stereo-PIV measurements perpendicular to the jet axis and assuming Taylor's hypothesis. They visualized groups of hairpin vortex structures in the fully developed turbulent region of jets in the range $1500<R e<5000$. Following the approach of Cintriniti and George, ${ }^{10}$ Jung et al. ${ }^{11}$ scanned the first 6 diameters of a turbulent axisymmetric jet at high Reynolds number with a polar array of 138 synchronized straight hot wire probes and showed a low-dimensional time-dependent reconstruction of the streamwise velocity using the dominant proper orthogonal decomposition (POD) modes. Reconstruction of the full-field streamwise velocity component using the dominant POD modes shows clearly the evolution of the flow with downstream position, from "volcano-type" eruptions to a "propeller-like" pattern. In a later study, Iqbal and Thomas ${ }^{12}$ achieved a three-components implementation of the POD and reported a helical vortex structure beyond the tip of the potential core.

Lynch and Thurow ${ }^{13}$ applied a novel three-dimensional light intensity visualization technique developed by Thurow and Satija ${ }^{14}$ to study the large-scale vortices in a jet at $R e=10000$. Instantaneous visualization of the transition region showed the rupture of jet centerline axial-symmetry, while POD analysis of the image intensity identified flapping motion and the combination between flapping and helical modes. These results, however, remained to be ascertained with a 3D kinematic analysis from velocity and vorticity fields.

The description of the dynamical behavior and the 3D patterns of flow structures requires timeresolved (TR), volume based techniques. Wernet ${ }^{15}$ applied TR PIV measurements on a high-speed jet to characterize the turbulence by means of space-time correlation functions. Based on a high speed scanning stereo PIV system (Bruecker and Althaus ${ }^{16}$ ), Hori and Sakakibara ${ }^{17}$ produced a sequence of instantaneous 3D vorticity field at 9 jet diameters downstream the nozzle of a jet at $R e$ $=1000$. With the application of tomographic PIV (Elsinga et al. ${ }^{18}$ ) the transitional incompressible jet flow at $R e=5000$ was investigated by Violato and Scarano ${ }^{19}$ who described the evolution of three-dimensional structures in circular and chevron nozzle configurations. For the circular jet, they reported a pulsatile motion due to the shedding and pairing of axisymmetric vortices followed by the growth of azimuthal instabilities (Yule ${ }^{6}$ Liepmann and Gharib ${ }^{7}$ ) and counter-rotating pairs of streamwise filaments (Dimotakis et al. ${ }^{20}$ Paschereit et al.,${ }^{21}$ and Ganapathisubramani et al. ${ }^{22}$ ). However, the limited axial extent of the measurement domain only allowed the descriptions of the earliest stage of the 3D regime onset leaving open questions on the mechanism of the annular mixing-layer collapse.

The use PIV in combination with aeroacoustic analogies is a relatively recent strategy to investigate the sources of acoustic noise. For a broader prospective on this novel methodology, the reader is referred to the review article by Morris. ${ }^{23}$ In a Mach 0.85 jet, Seiner ${ }^{24}$ characterized the 
noise sources using two-point turbulence statistics to evaluate the Lighthill turbulent stress tensor and observed that the actual measurement of the Lighthill's acoustic tensor requires volumetric measurements with temporal resolution. In a subsonic and acoustically excited jet flow at low Reynolds number, Schram et al. ${ }^{25}$ investigated the mechanism of sound generation by vortex pairing with phase-locked planar PIV and a conservative formulation of vortex sound theory for axisymmetric flow, finding good agreement between the sound prediction and the sound measured by microphone at frequencies not contaminated by the acoustical excitation. Fleury et al. ${ }^{26}$ used a dual-PIV system in a subsonic jet at Mach 0.6 and 0.9 with a fixed time delay between measurements to calculate spatial and temporal correlations. They also showed that Lighthill stress tensor can be statistically modelled by space-time second-order velocity correlations. Violato and Scarano ${ }^{19}$ first used timeresolved three-dimensional measurements to explore the relation between the coherent structures and the instantaneous acoustic production based on Powell's analogy. ${ }^{27}$ The acoustic source was characterized by the second time derivative of the Lamb vector while the corresponding vortex topology was visualized using $\lambda_{2}$-criterion (Jeong and Hussain ${ }^{28}$ ). In the region near-to-the-nozzle exit of the circular jet, the acoustic source activity was highly correlated with vortex-ring pairing and the growth of azimuthal instabilities contoured by streamwise filaments. However, performing acoustic predictions based on Powell's integral is a challenging task, especially due to the constraints on the extent of the instantaneous measurement domain and on the required spatial and temporal resolutions. For this reason, acoustic analyses have been mostly afforded using direct numerical simulations (DNS; Freund ${ }^{29}$ ) and large eddy simulations (LES; Bogey et al. ${ }^{4}$ ) techniques to obtain accurate descriptions of the flow field.

In the present work, a state-of-the-art measurement system is employed to perform TR-TOMO PIV measurements over a jet axial extent that is sufficiently long (10 jet diameters) to capture the large-scale events from the nozzle to 4 jet diameters beyond the end of the potential core. The experiments therefore enable the analysis of the jet core breakdown with the spatial and temporal resolution (up to a value of the Strouhal number of 4) needed for the evaluation of Powell's integrand. The investigation is conducted under favorable experimental conditions in a water facility, where a relatively large measurement domain can be accessed with TOMO-PIV (Scarano and Poelma ${ }^{30}$ ) and where high-speed PIV systems can operate at a repetition such to resolve the smallest flow time scales.

The first objective of this study is to analyze the three-dimensional evolution of vortex rings during the process of breakdown into coherent substructures. The corresponding unsteady 3D patterns are described using the vorticity vector components and the $\lambda_{2}$-criterion, which, experimentally, could not be fully ascertained by planar PIV (Liepmann and Gharib, ${ }^{7}$ Dimotakis et al.,${ }^{20}$ Ganapathisubramani et al. ${ }^{22}$ Matsuda and Sakakibara, ${ }^{9}$ and Wernet ${ }^{15}$ ), visualization techniques (Yule, ${ }^{6}$ Kyle and Shreenivasan, ${ }^{8}$ and Lynch and Thurow ${ }^{13}$ ), or by arrays of hot wire probes (Jung et al. ${ }^{11}$ ). Following Powell's analogy, the relation between large-scale events and acoustic source is associated with the second time derivative of the Lamb vector. The analysis of the 3D pattern of velocity, vorticity, Lamb-vector and second time derivative of the Lamb vector is aided by proper orthogonal decompositions, which enable to identify more clearly some aspects of the flow large-scale organization at core breakdown. Finally, acoustic predictions are performed by direct integration Powell's analogy using TR-TOMO PIV data.

\section{SNAPSHOT POD ANALYSIS}

Proper orthogonal decomposition (Berkooz et al. ${ }^{31}$ ) is a statistical technique to objectively classifying and describing turbulent flows in terms of most energetic coherent motions that can be used to produce of a low-order reconstruction of the flow field. When applied to velocity fields, such as PIV data or numerical simulations, POD analysis enables the identification of the coherent structures in terms of global eigenmodes. POD was applied to planar PIV data to describe a large variety of jet flow configurations: annular jets (Patte-Rouland et $a l .{ }^{32}$ ), normally impinging jets (Geers et al. ${ }^{33}$ ) turbulent jets in crossflow (Meyer et al. ${ }^{34}$ ), daisy- and cross-shaped orifice jets (El Hassan and Meslem and ${ }^{35}$ El Hassan et al. $\left.{ }^{36}\right)$. More recently, Schmid et al. ${ }^{37}$ have investigated the use of dynamical mode decomposition (DMD) for the analysis of TR-TOMO PIV data of the same 
jet. Although, the results show that DMD is a suitable technique to infer the dynamical features of the velocity fluctuations, the present work will be based on POD also for reasons of comparison with the more abundant literature available.

The POD principles are briefly introduced here following the method of snapshots $\left(\operatorname{Sirovich}^{38}\right)$. Consider a set of data $u\left(\mathbf{x}, t_{n}\right)$ that are simultaneously taken at $N$ time instants such that the samples are uncorrelated and linearly independent. The corresponding fluctuating component is defined as

$$
u^{\prime}\left(\mathbf{x}, t_{n}\right)=u\left(\mathbf{x}, t_{n}\right)-\bar{u}\left(\mathbf{x}, t_{n}\right),
$$

where $\bar{u}\left(\mathbf{x}, t_{n}\right)$ is the temporal average

$$
\bar{u}\left(\mathbf{x}, t_{n}\right)=\frac{1}{N} \sum_{n=1}^{N} u\left(\mathbf{x}, t_{n}\right) .
$$

The POD method extracts orthonormal eigenmodes $\psi(\mathbf{x})$ and orthonormal amplitude coefficients $a_{k}(t)$ such that the reconstruction

$$
u^{\prime}\left(\mathbf{x}, t_{n}\right)=\sum_{k=1}^{\infty} a_{k}(t) \psi_{k}(\mathbf{x})
$$

is optimal, in the sense that the functions $\psi$ maximize the normalized average projection of $\psi$ onto $u^{\prime}$

$$
\max _{\psi} \frac{\left\langle\left(\psi(\mathbf{x}), u^{\prime}(\mathbf{x}, t)\right)^{2}\right\rangle}{(\psi(\mathbf{x}), \psi(\mathbf{x}))} .
$$

The time coefficients $a_{k}(t)$ are determined by the projection of the flow-fields on the global modes

$$
a_{k}(t)=\left(u^{\prime}(\mathbf{x}, t), \psi_{k}(\mathbf{x})\right) .
$$

The snapshot method introduced by Sirovich $^{38}$ is less computationally demanding and the above maximization problem corresponds to solve a degenerate integral equation, in which the solutions are linear combinations of the snapshots

$$
\psi_{k}(\mathbf{x})=\sum_{n=1}^{N} \Phi_{n}^{k} u^{\prime}\left(\mathbf{x}, t_{n}\right) \quad k=1, \ldots, N
$$

where $\Phi_{n}^{k}$ is the $n$th component of the $k$ th eigenvector. The eigenmodes can then be found by solving the following eigenvalue problem

$$
C_{u} \Phi=\lambda \Phi
$$

where $C$ is the $L_{2}$-norm matrix,

$$
C_{u}=\frac{1}{N}\left(u^{\prime}(\mathbf{x}), u^{\prime}(\mathbf{x})\right) .
$$

The cumulative sum of the eigenvalues $\lambda_{k}$ corresponds to the total energy and each eigenmode is associated with an energy percentage $e_{k}$ :

$$
e_{k}=\lambda_{k} / \sum_{i=1}^{N} \lambda_{i}
$$

POD descriptions of PIV data are commonly produced choosing the mean square fluctuating velocity as norm, which represents the kinetic energy of the flow. However, a more efficient identification of the coherent structures in PIV data can be obtained by the vorticity decomposition, as proposed by Kostas et al., ${ }^{39}$ who applied POD on the velocity and the magnitude of the out-of-plane vorticity. Such quantity, as explained by the authors, is a quasi-enstrophy, since only one component of the vorticity was considered. 
In this investigation, POD analysis is first conducted on three-dimensional velocity data sets based on the mean square fluctuating energy (Eq. (1)), which, for the fluctuating velocity vector $\left(\mathbf{V}^{\prime}\right)$ can be written as

$$
C_{V}=\frac{1}{N}\left(\mathbf{V}^{\prime}(\mathbf{x}), \mathbf{V}^{\prime}(\mathbf{x})\right) .
$$

Then, by centered difference scheme, the velocity information is used to evaluate the threedimensional vorticity field. This is analyzed by POD using, as norm, the mean square fluctuating enstrophy

$$
C_{\omega}=\frac{1}{N}\left(\omega^{\prime}(\mathbf{x}), \omega^{\prime}(\mathbf{x})\right),
$$

where $\boldsymbol{\omega}^{\prime}$ is the fluctuating vorticity vector.

Moreover, the velocity and the vorticity vectors are used to evaluate the Lamb vector $\mathbf{L}=\boldsymbol{\omega} \times \mathbf{V}$, which is a relevant quantity in aeroacoustics as it accounts for the flow state in Powell's aeroacoustic analogy (see the Appendix). The modal analysis of the Lamb vector field is conducted by POD based on the norm of the fluctuating Lamb vector $\mathbf{L}^{\prime}$

$$
C_{L}=\frac{1}{N}\left(\mathbf{L}^{\prime}(\mathbf{x}), \mathbf{L}^{\prime}(\mathbf{x})\right),
$$

which will be referred to as mean square fluctuating Lamb energy. Finally, following Powell's aeroacoustic analogy, the acoustic source is related to the second time derivative of the Lamb vector $d^{2} \mathbf{L} / d t^{2}$ (see the Appendix), which is analyzed with the POD technique based on the norm of the fluctuating component $d^{2} \mathbf{L} / d t^{2 \prime}$

$$
C_{d^{2} \mathbf{L} / d t^{2}}=\frac{1}{N}\left(d^{2} \mathbf{L} / d t^{2 \prime}(\mathbf{x}), d^{2} \mathbf{L} / d t^{2 \prime}(\mathbf{x})\right) .
$$

The above norm will be referred to as mean square fluctuating acoustic source energy.

\section{EXPERIMENTAL APPARATUS AND PROCEDURES}

\section{A. Jet flow facility}

Experiments are conducted in the water jet facility described in Violato and Scarano ${ }^{19}$ at the Aerodynamic Laboratories of TU Delft in the Aerospace Engineering Department. A round nozzle of exit diameter $D=10 \mathrm{~mm}$ and contraction ratio of 56:1, is installed at bottom wall of an octagonal water tank. The system is hydrostatically driven and provides a stabilized supply in a range of exit velocity $W_{j}$ from 0.1 to $2 \mathrm{~m} / \mathrm{s}$, corresponding to Reynolds numbers ranging between 1000 and 20000 . The water temperature is $20^{\circ} \mathrm{C}$. Tomographic experiments are performed for a nominal axial velocity at the jet exit of $0.5 \mathrm{~m} / \mathrm{s}$ yielding a Reynolds number $R e=5000$ based on the jet diameter $D$.

\section{B. Time-resolved tomographic measurements}

Neutrally buoyant polyamide particles of $56 \mu \mathrm{m}$ of diameter are dispersed homogeneously, achieving a uniform concentration of 0.65 particles $/ \mathrm{mm}^{3}$. The illumination is provided by a Quantronix Darwin-Duo solid-state diode-pumped Nd:YLF laser $(2 \times 25 \mathrm{~mJ} /$ pulse at $1 \mathrm{kHz})$. After a transmission distance of $1.5 \mathrm{~m}$, the laser beam features a diameter of $6 \mathrm{~mm}$ and is further expanded through a diverging lens to a diameter of $80 \mathrm{~mm}$ and then focused with a converging lens to obtain a conical illumination domain (Figure 1). The light scattered by the particles is recorded by a tomographic system composed of three 3 Imager pro HS 4M cameras (12 bits, $2048 \times 2048$ pixels, pixel pitch of $11 \mu \mathrm{m}$ ) arranged horizontally with azimuthal aperture of $90^{\circ}$. The choice of 
TABLE I. Experimental settings for time-resolved measurements.

\begin{tabular}{ll}
\hline \hline Seeding material & Polyamide particles diameter: $56(\mu \mathrm{m})$ \\
& Concentration : $0.65\left(\right.$ particles $\left./ \mathrm{mm}^{3}\right)$ \\
Illumination & Quantronix Darwin-Duo Nd-YLF laser $(2 \times 25 \mathrm{~mJ} @ 1 \mathrm{kHz})$ \\
Recording device & 3 Imager pro HS 4M cameras $(2016 \times 2016$ pixels@ $1.3 \mathrm{kHz}) 11 \mu \mathrm{m}$ pixel pitch \\
Recording method & double frame $/$ single exposure continuous mode \\
Optical arrangement & Nikon objectives $(\mathrm{f}=105 \mathrm{~mm} ; \mathrm{f}=22)$ field of view 5D $\times 10 \mathrm{D}$ \\
Acquisition frequency & $1 \mathrm{kHz}$ \\
Time of acquisition & $2 \mathrm{~s}$ \\
\hline \hline
\end{tabular}

conical illuminated volume eliminates the need for camera-lens tilt mechanism to comply with the Scheimpflug condition. Nikon objectives of $105 \mathrm{~mm}$ focal length are set with a numerical aperture $f_{\#}=22$ to allow focused imaging of the illuminated particles. For the chosen illumination and imaging configuration the particle image density decreases from the axis towards the edge of the illuminated volume and, at the jet axis, increases along the axial coordinate from 0.037 (at the jet exit) to 0.043 ( 10 diameters above the exit) particles/pixel. The details of the experimental settings are summarized in Table I.

The choice of a conical domain of illumination more favors an accurate reconstruction of the particle field, as the particle image density does not change with the viewing angle along the azimuth and decreases moving from the axis to the periphery of the jet (Figure 1). Moreover, the extent of uniform flow velocity in the region outside the jet is minimized, which mitigates the effect of ghost particles velocity (Elsinga et al. ${ }^{40}$ ) in turn biasing the measurement in the shear layer and jet core (Novara and Scarano ${ }^{41}$ ).

Sequences of images of tracer particles are recorded at $1 \mathrm{kHz}$ resulting in a temporal resolution that yields 35 samples for the fastest expected events (vortex shedding). The imaged-particle displacement at the exit is approximately 10 pixels along the jet axis. The field of view is of $50 \times 100 \mathrm{~mm}$ with a digital resolution of 18.5 pixels $/ \mathrm{mm}$. Sequences of 500 images are also recorded at $20 \mathrm{~Hz}$ in frame-straddling mode (pulse separation time $1 \mathrm{~ms}$ ) for a total time of $25 \mathrm{~s}$ to produce a statistically significant data ensemble.

The volumetric light intensity reconstruction is performed following the use of multiplicative algebraic reconstruction technique algorithm (MART, Hermann and Lent ${ }^{42}$ ) by the LaVision software DAVIS 8. A three-dimensional mapping function from image-space to physical object-space is
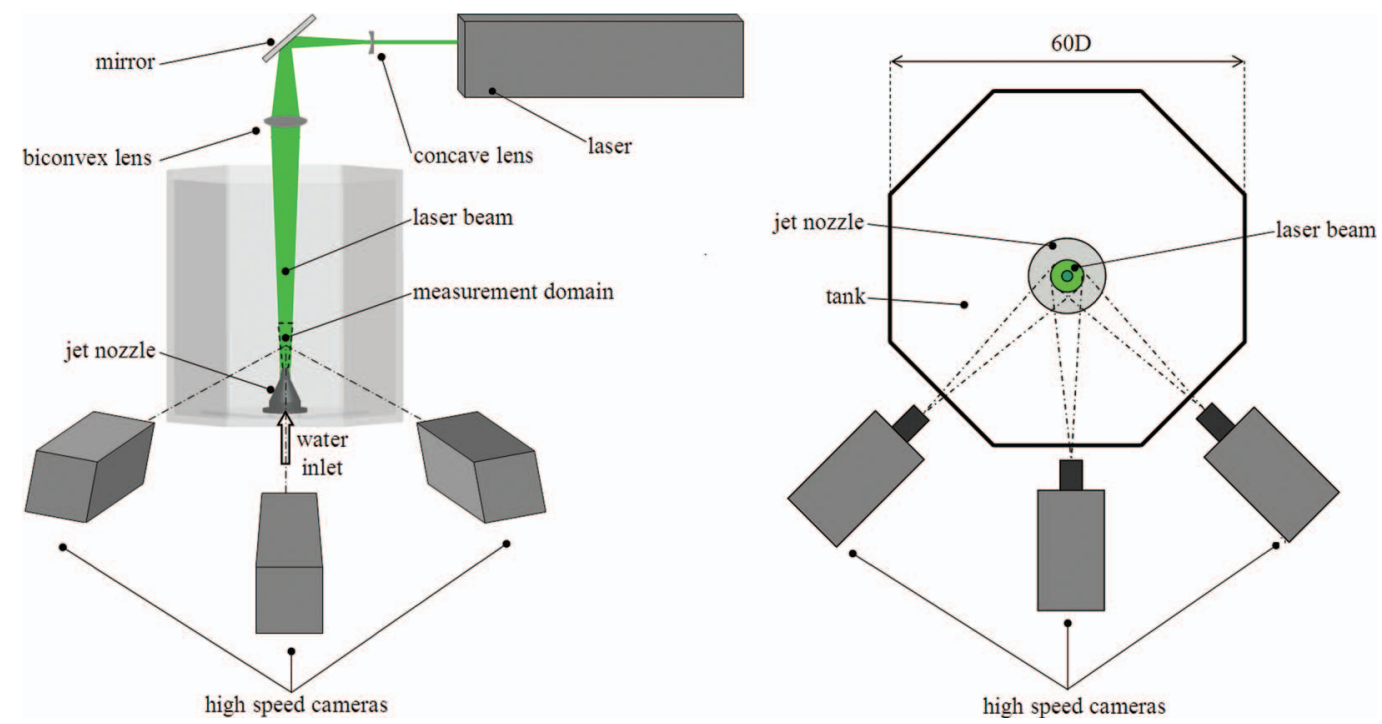

FIG. 1. Schematic view of illumination and imaging in the tomographic experiment (left); top view of the system (right). 


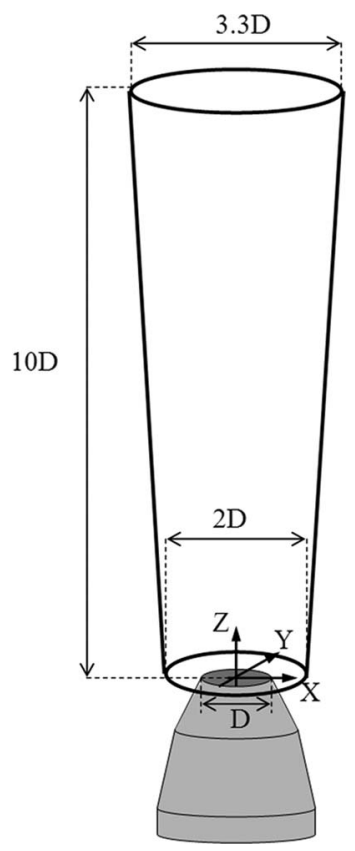

FIG. 2. Details of the measurement domain and coordinate system.

generated by imaging a calibration target. The initial experimental errors due to system calibration are approximately 0.5 pixels as estimated from the disparity vector field. The misalignment is reduced to less than 0.05 pixels making use of the $3 D$ self-calibration technique (Wieneke ${ }^{43}$ ). The raw images are pre-processed with subtraction of the minimum intensity at each pixel for the entire sequence, followed by a subtraction of the local minimum over a kernel of $31 \times 31$ pixels. The MART algorithm is applied with four iterations. At the given particle image density, despite the radial decrease and axial increase, the use of 3-camera tomographic system leads to rather accurate 3D object reconstructions with a reconstruction quality $Q$ estimated above 0.75 (Elsinga et al. ${ }^{18}$ ).

The illuminated volume is discretized with $660 \times 660 \times 2000$ voxels resulting in a digital resolution of 20 voxels $/ \mathrm{mm}$ (voxel pitch of $50 \mu \mathrm{m}$ ). Following Elsinga et al. ${ }^{18}$ the accuracy of the reconstruction is evaluated a posteriori comparing the intensity of reconstructed particles in the illuminated region with that of ghost particles produced in the immediate surroundings of the illuminated domain. A signal-to-noise ratio may be defined as the reconstructed particles intensity inside the illuminated area versus that reconstructed outside. In the present experiment, the laser light intensity follows a Gaussian distribution along the radius and the light is confined to the conical volume shown in Figure 2. The reconstruction signal-to-noise ratio as defined above is higher than 4.

The three-dimensional particle field motion is computed by volume deformation iterative multigrid (VODIM) technique (Scarano and Poelma ${ }^{30}$ ) with a final interrogation volume of $40 \times 40 \times 40$ voxels $\left(2 \times 2 \times 2 \mathrm{~mm}^{3}\right)$ with an overlap between adjacent interrogation boxes of $75 \%$, leading to a vector pitch of $0.5 \mathrm{~mm}$. Correlation averaging technique over three subsequent object-pairs is applied to have higher signal-to-noise ratio and higher measurement precision in time-resolved measurements $\left(\mathrm{Scarano}^{44}\right)$. Data processing is performed on a dual quad-core Intel Xeon processor at $2.83 \mathrm{GHz}$ with $8 \mathrm{~GB}$ RAM memory requiring, respectively, $1 \mathrm{~h}$ and $40 \mathrm{~min}$ for the reconstruction of a pair of objects and $2 \mathrm{~h}$ for the $3 \mathrm{D}$ cross-correlation.

When needed, data post-processing is applied that consists of a space-time least-squares regression. Spatio-temporal noisy fluctuations are significantly suppressed by applying the second order polynomial least squares regression (Scarano and Poelma ${ }^{30}$ ) with a kernel of $5 \times 5 \times 5$ grid nodes in space $\left(2 \mathrm{~mm}^{3}\right)$, corresponding to the interrogation box size. Given the high repetition rate of the measurement a temporal kernel that encompasses 7 time steps $(6 \mathrm{~ms})$ is used without affecting the 


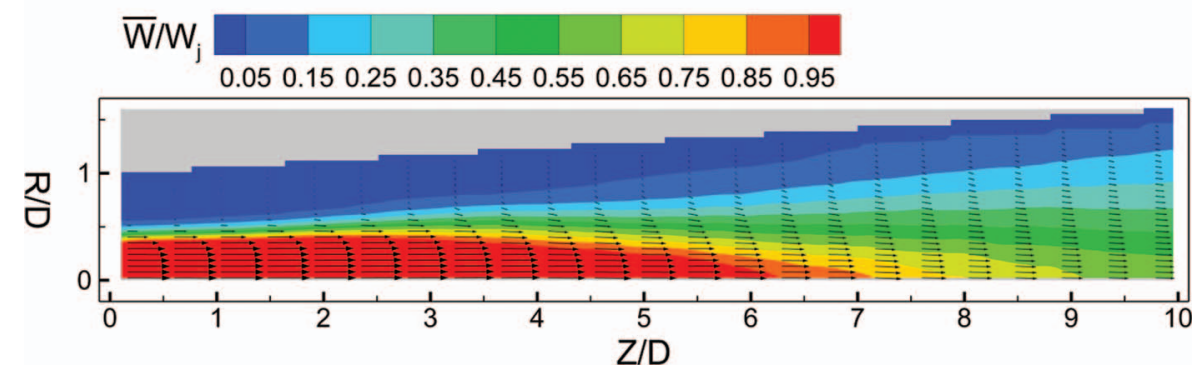

FIG. 3. Iso-contours of mean-axial velocity on a radial plane.

temporal resolution of the measurement in the range of time scales of interest (up to a value of the Strouhal number of 4). The precision error on the filtered velocity and vorticity fields are estimated following Violato and Scarano. ${ }^{19}$ It results that the velocity is affected by $2 \%$ precision error with respect to the particle displacement at the jet exit of 10 voxels; the error on vorticity is estimated to be $3 \%$ with respect to the vorticity magnitude at the core of a shed vortex ( 0.25 voxels/voxel). For details on the correlation technique, filtering method and spatio-temporal resolution of the data, the reader is referred to Violato and Scarano. ${ }^{19}$

\section{RESULTS}

\section{A. Flow statistics}

The spatial distribution of the mean axial velocity is illustrated in Figure 3 as iso-contours on a radial plane on the cylindrical domain. The potential core, which is identified by the contour line $\bar{W} / W_{j}=0.95$, shows an axial penetration of 6.25 diameters. Further downstream, the mean axial velocity decreases to 0.55 at $Z / D=10$ (Crow and Champagne ${ }^{45}$ ). The detailed characterization of the mean velocity profile at the exit, as well as the level of axial velocity fluctuations in the jet core, are reported in Violato and Scarano. ${ }^{19}$

The peak activity of the turbulent fluctuations is concentrated between $Z / D=2.5$ and Z/D $=8.5$, as depicted by the iso-contour plots of Figure 4. For $2.5<\mathrm{Z} / \mathrm{D}<4.5$, axial and radial fluctuations $w^{\prime} / W_{j}$ and $v_{r}^{\prime} / W_{j}$ reach peaks of 0.18 , where the vortex rings pair (Bradshaw et al ${ }^{46}$ and Yule $^{6}$ ) and azimuthal instabilities and streamwise vortices grow (Violato and Scarano ${ }^{19}$ ). The azimuthal fluctuations $v_{\theta}^{\prime} / W_{j}$, instead, are dominant in the region by the end of the potential core. For low Reynolds number jets, Yule ${ }^{6}$ reported that circumferential fluctuations intensify as the flow three-dimensionality develops and the axisymmetric regime vanishes rapidly.

\section{B. Unsteady flow behavior}

The instantaneous flow organization is depicted in Figure 5. Toroidal vortices are clearly identified by iso-surfaces of azimuthal vorticity $\omega_{\theta} D / W_{j}$ whereas the three-dimensional flow structures are visualized by the axial and radial vorticity components $\omega_{z} D / W_{j}$ and $\omega_{r} D / W_{j}$. The separate visualization of each component of the vorticity vector enables a more direct evaluation of the flow organization. In the region near to the nozzle exit $(Z / D=0$ and $Z / D=5.5)$, the flow exhibits pulsatile motion (iso-surface $W / W_{j}$ ) driven by the shedding and pairing of toroidal vortices at Strouhal number $S t=f D / W_{j}=0.72$ and 0.36 , respectively. Vortex pairing is accompanied by to the growth of azimuthal instabilities and the formation of counter-rotating pairs of streamwise vortices of radial and axial vorticity (Violato and Scarano ${ }^{19}$ ) that surround the jet shear layer. In the region beyond the end of the potential core $\left(Z / D>6\right.$, Figure 3 ) vortex ring coherence is absent (Yule ${ }^{6}$ and Hussain and $Z a m a n^{2}$ ) and the flow is mainly organized into streamwise vortex filaments. Liepmann and Gharib ${ }^{7}$ reported that the streamwise vorticity is the main factor controlling the entrainment process beyond the end of the potential core. 

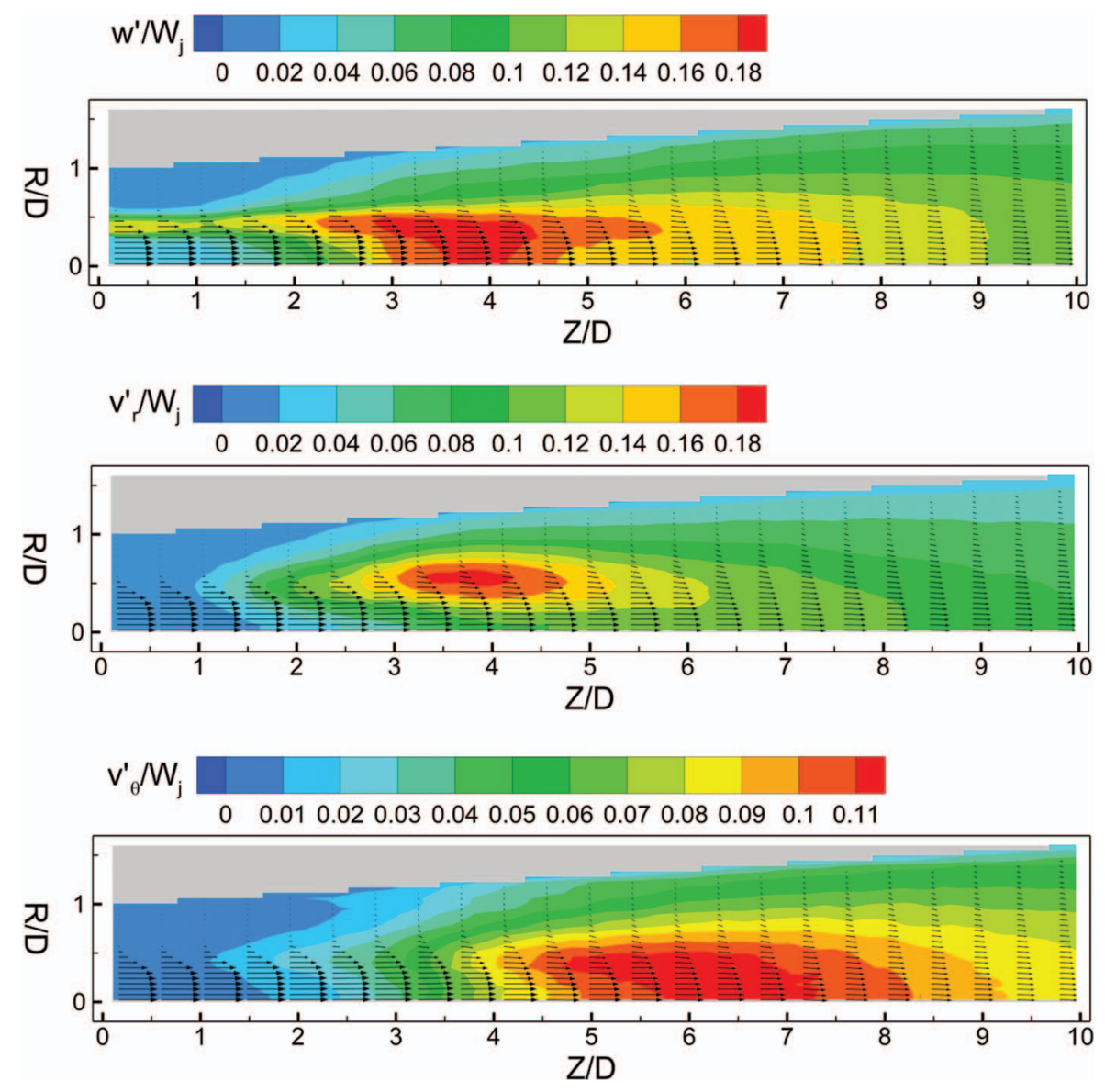

FIG. 4. Iso-contours of velocity fluctuations on a radial plane: axial $w^{\prime} / W_{j}$, radial $v_{r}^{\prime} / W_{j}$ and tangential $v_{\theta}^{\prime} / W_{j}$ components.

The spatio-temporal evolution of a selected event during the breakdown of a toroidal vortex is shown in the temporal sequence of Figure 6 where the iso-surface $\lambda_{2} D / W_{j}=-0.8$ identifying the vortices are color-coded by the instantaneous axial velocity $W / W_{j}$. To facilitate the visualization, iso-surfaces are mapped between $R / D=0$ and $R / D=1$. The decay of the azimuthal coherence of toroidal structures begins after pairing (Violato and Scarano ${ }^{19}$ ) with the growth of four in-plane (see label "P", $t W_{j} / D=0$ ) and four out-of-plane azimuthal waves. With the growth of in-plane azimuthal instabilities, portions of the vortex ring (see labels "P") are tilted inward and stretched towards the jet axis $\left(0<t W_{j} / D<1.26\right)$, where the axial velocity is larger $\left(W / W_{j}=1\right)$. Due to the asymmetric distribution of $W$, regions "P" are then ejected and tilted along the jet axis $(1.68$ $\left.<t W_{j} / D<2.1\right)$, producing axial stretching and, ultimately, the disruption of the toroidal shape (Z/D $=5.8$ and 6.5). Bogey et al. ${ }^{4}$ associated such transient event to a possible mechanism of acoustic generation. By the end of the potential core, the characteristic axial and radial velocity fluctuations are $w^{\prime} / W_{j}=0.16$ and $v_{r}^{\prime} / W_{j}=0.14,30 \%$ smaller than those observed for the pairing process (Figure 4). In contrast, azimuthal velocity component reaches peak activity of 0.11 , which is about twice that at pairing. Vortex portions originated from low-axial velocity regions gradually disrupt into smaller structure of "C" shape (see label "C", $1.68<t W_{j} / D<2.52$ ), whereas those originated from region "P" form an angle of $30^{\circ}-45^{\circ}$ with the jet axis $\left(2.52<t W_{j} / D<2.94\right)$. The evolution of the breakdown hereby reported agrees with the early conjectures by Hussain and Zaman ${ }^{2}$ who described that toroidal vortices develop azimuthal lobes and disrupt into coherent substructures by the end of the potential core.

Downstream the potential core $(7<Z / D<10)$, the three-dimensional regime exhibits, as most recurrent pattern, vortex filaments with scattered orientation (Figure 7). The decay of the vortex 

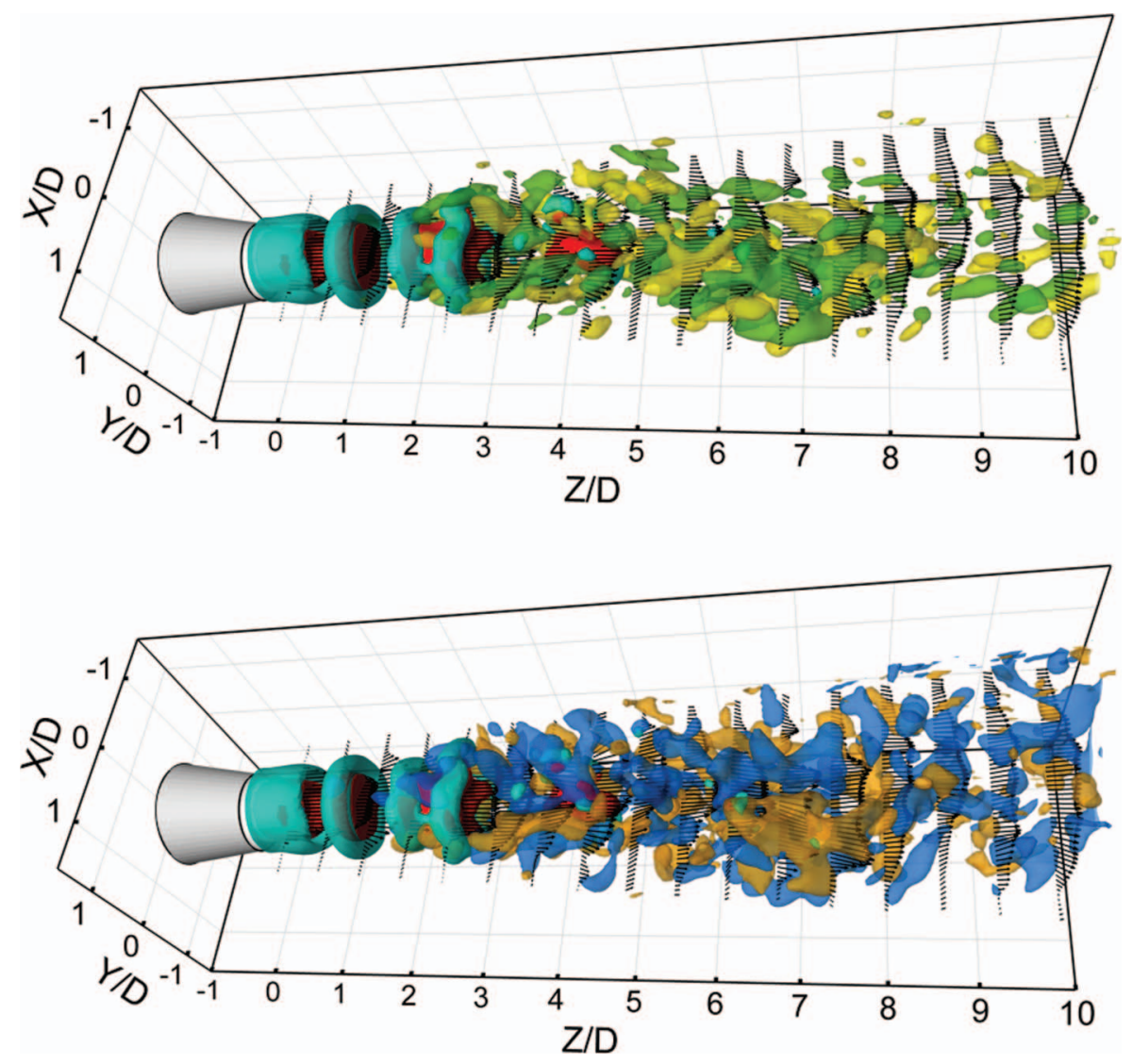

FIG. 5. Instantaneous vortex pattern in the circular jet. Velocity vectors are shown on the axial plane $Y / D=0$. Iso-surfaces of axial velocity $W / W_{j}=1.05$ (red). Top: iso-surfaces $\omega_{\theta} D / W_{j}=4$ (cyan), $\omega_{z} D / W_{j}=-1.2$ (green) and 1.2 (yellow); bottom: iso-surfaces $\omega_{\theta} D / W_{j}=4$ (cyan), $\omega_{r} D / W_{j}=-1.2$ (blue) and 1.2 (orange).

coherence is faster between $0<R / D<0.8$ where the axial velocity is characterized by flow patches of maximum axial velocity $W / W_{j}=0.6$, and velocity fluctuations $w^{\prime} / W_{j}, v_{r}^{\prime} / W_{j}$ and $v_{t}^{\prime} / W_{j}$ of comparable magnitude (0.1-0.12, Figures 4 and 3$)$. By contrast, in the periphery of the jet $(0.8<R / D$ $<1.6)$, where fluctuations are about $50 \%$ smaller, "C" structures $\left(t W_{j} / D=0\right)$ tend to re-organize into filaments "VF" $\left(t W_{j} / D=1.68\right)$. "VF" structures are typically oriented along the azimuthal direction and have a characteristic length ranging between 1 and 2 jet diameters, based on iso-surface $\lambda_{2} D / W_{j}$ $=-0.5$. The advection velocity along the axial direction is 0.2 . As shown in Figures $7(\mathrm{c})$ and $7(\mathrm{~d})$, they engulf outer fluid on the trailing side and eject on the leading one, with peaks of instantaneous radial velocity of 0.1 (Yule, 1978). The typical lifetime of the "VF" is $\Delta t W_{j} / D=6$ after which they break-up into sub-structures "VFsub" $\left(t W_{j} / D=6.3\right)$ between $Z / D=9$ and 10 . Yule ${ }^{6}$ reported the presence of vortices with similar patterns up to 5 jet diameters downstream the transition.

The normalized axial, radial, and tangential flux associated with the "VF" structure can be defined as

$$
\left\{Q_{w}, Q_{r}, Q_{\theta}\right\}=\frac{1}{D^{2}} \int_{S}\left\{W / W_{j} \hat{e}_{w}, V_{r} / W_{j} \hat{e}_{r}, V_{\theta} / W_{j} \hat{e}_{\theta}\right\} \cdot \overline{d S},
$$

where $\mathrm{S}$ is the surface of the volume containing the "VF" vortex, $\hat{e}_{w}, \hat{e}_{r}$, and $\hat{e}_{\theta}$ denote the unit vector in the axial, radial, and tangential direction, $\overline{d S}$ is the vector normal to $S$. On average, the flux related with "VF" shows that the axial and the radial components have the same magnitude and opposite sign $\left(Q_{w}=0.075 ; Q_{r}=-0.075\right)$, whereas the tangential component is one order magnitude smaller. Compared to Liepmann and Gharib, ${ }^{7}$ who ascribed to streamwise vorticity the major role in the entrainment of the flow beyond the end of the potential core, the observations 
(a)

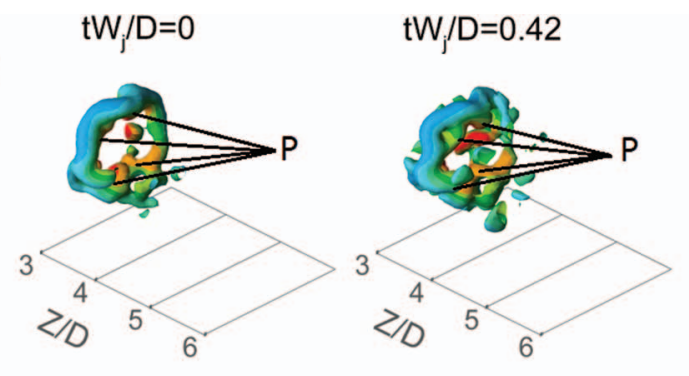

$$
\mathrm{tW}_{\mathrm{j}} / \mathrm{D}=0.84 \quad \mathrm{t} \mathrm{W}_{\mathrm{j}} / \mathrm{D}=1.26
$$

(b)

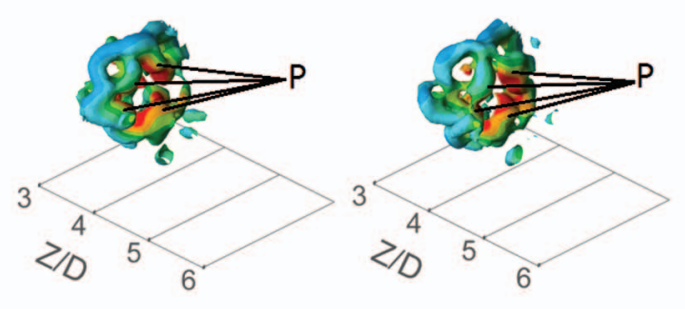

$$
\mathrm{tW} / \mathrm{D}=1.68
$$

\section{$\mathrm{tW}_{\mathrm{j}} / \mathrm{D}=2.1$}

(c)
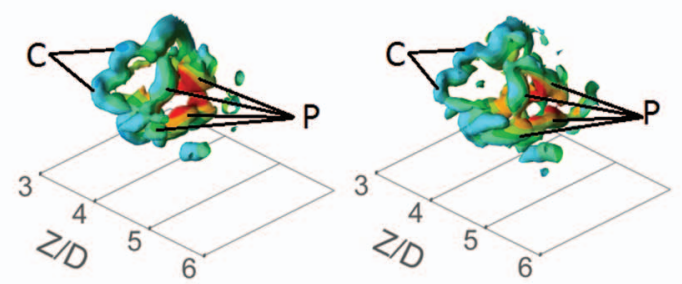

(d)
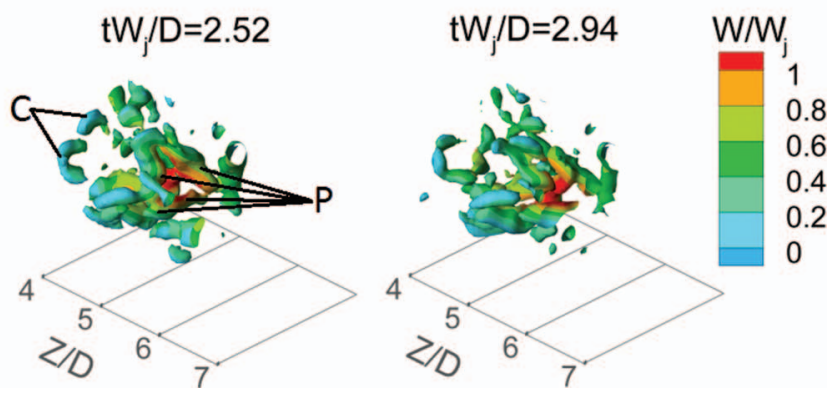

FIG. 6. Time sequence visualization of vortex ring breakdown. Iso-surface $\lambda_{2} D / W_{j}=-0.8$ color coded with axial velocity component $W / W_{j}$. Time separation between snapshots $\Delta t W_{j} / D=0.42$. (a) Azimuthal waves inception. (b) Induced axial velocity. (c) Breakdown of azimuthal coherence. (d) Turbulent engulfment.

on "VF" filaments hereby reported provide a more specific characterization of the vortex pattern involved in such entrainment.

An overview on the flow transition to three-dimensionality along the jet axis can be inferred from the axial distributions of the vorticity components, as illustrated in Figure 8 where the absolute values of the azimuthal, the radial and the axial components $\left(\left|\omega_{\theta} D / W_{j}\right|,\left|\omega_{r} D / W_{j}\right|,\left|\omega_{z} D / W_{j}\right|\right)$ are averaged in time and along the azimuthal direction between $R / D=0$ and $R / D=1,\left\langle\left|\omega_{\theta} D / W_{j}\right|\right\rangle$, $\left\langle\left|\omega_{r} D / W_{j}\right|\right\rangle$ and $\left\langle\left|\omega_{z} D / W_{j}\right|\right\rangle$. Downstream the shedding and pairing of toroidal structures $(Z / D$ $>3.5),\left\langle\left|\omega_{r} D / W_{j}\right|\right\rangle$ and $\left\langle\left|\omega_{z} D / W_{j}\right|\right\rangle$ increase as azimuthal instabilities and streamwise vortices 


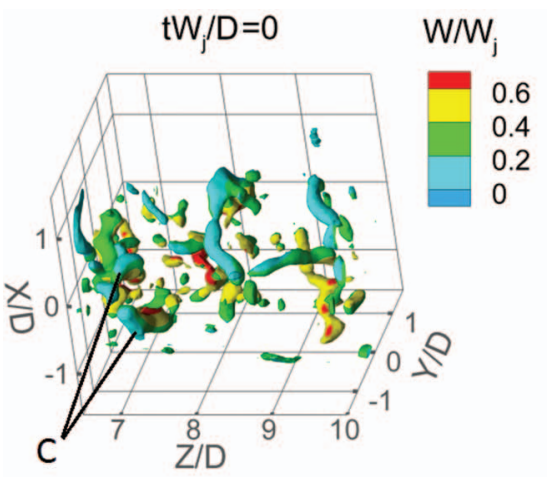

(a)

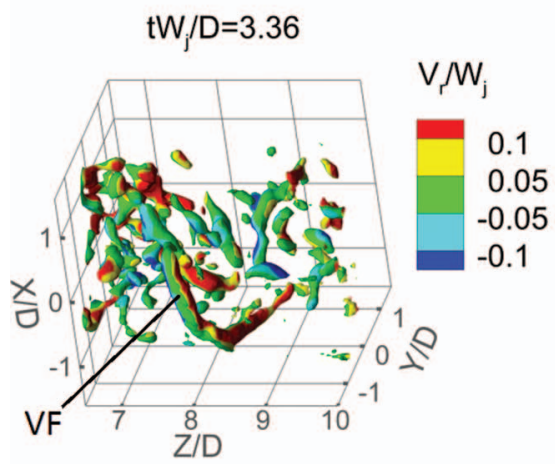

(c)

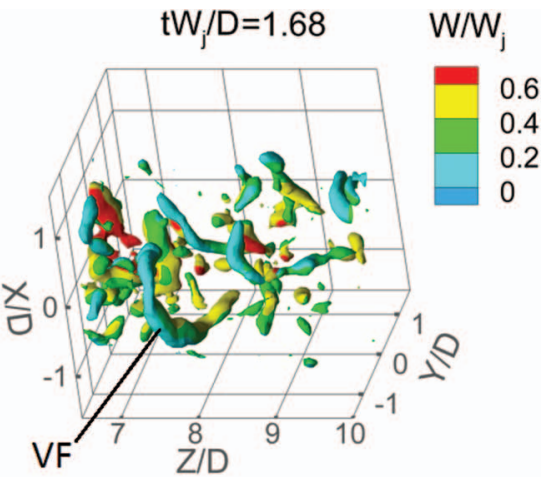

(b)

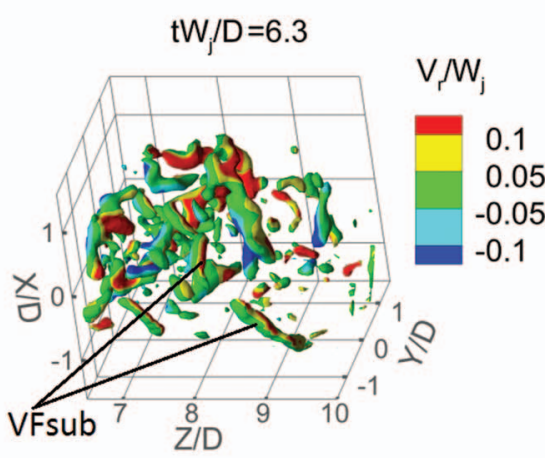

(d)

FIG. 7. Time sequence visualization of vortices after breakdown. Phenomenon of $C$-vortex connect and subsequent filament tearing. Iso-surface $\lambda_{2} D / W_{j}=-0.5$ color coded with $W / W_{j}$ (a) and (b) and $V_{r} / W_{j}$ (c) and (d). (a) C-structures. (b) Vortex filament formation. (c) Vortex filament advection. (d) Vortex filament breakdown.

grow, while the azimuthal component $\left\langle\left|\omega_{\theta} D / W_{j}\right|\right\rangle$ is still dominant although steadily decreasing. In the region near the end of the potential core $(5.5<Z / D<6.5),\left\langle\left|\omega_{z} D / W_{j}\right|\right\rangle$ reaches a peak $(0.63$ at $Z / D=5.5)$ corresponding with the ejection and axial tilting of the "P" flow regions (Figure 6). In contrast, $\left\langle\left|\omega_{r} D / W_{j}\right|\right\rangle$ steadily increases to 0.73 at $Z / D=6.2$, where the toroidal structures breakdown. Beyond the potential core $(Z / D>6.5)$, the flow field shows an overall

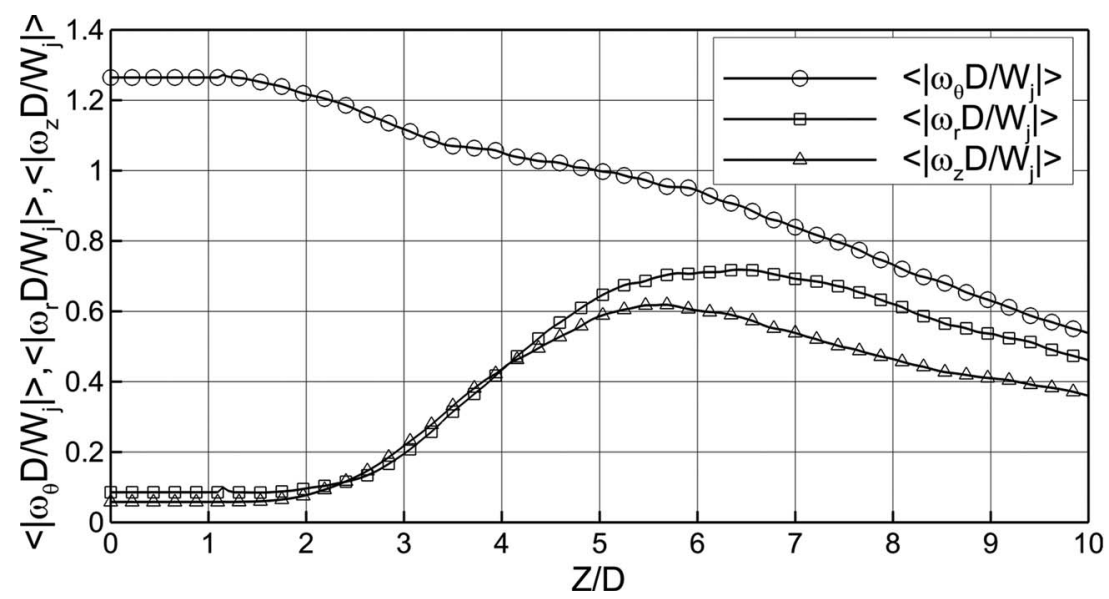

FIG. 8. Axial distribution of $\left\langle\left|\omega_{\theta} D / W_{j}\right|\right\rangle,\left\langle\left|\omega_{r} D / W_{j}\right|\right\rangle$ and $\left\langle\left|\omega_{z} D / W_{j}\right|\right\rangle$. 

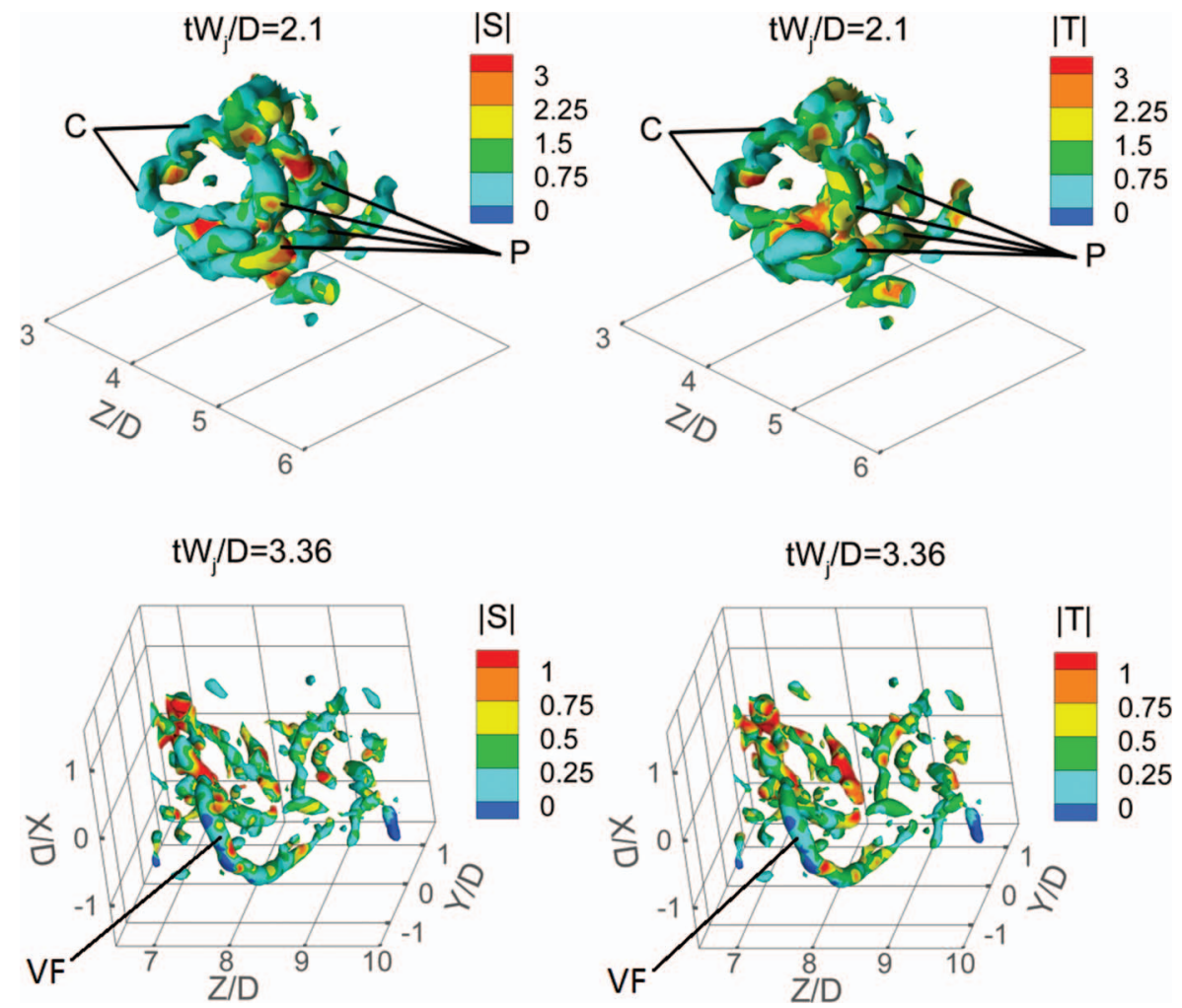

FIG. 9. Instantaneous stretching and tilting fields. Top: vortex breakdown identified by iso-surfaces $\lambda_{2} D / W_{j}=-0.8$ color coded with $|\mathrm{S}|$ and $|\mathrm{T}|$; bottom: "VF" vortices identified by iso-surfaces $\lambda_{2} D / W_{j}=-0.8$ color coded with $|\mathrm{S}|$ and $|\mathrm{T}|$.

decrease of the vorticity components to values comparable to those in the region of growth of azimuthal instabilities and streamwise vortices formation (Z/D 4).

The vortex stretching-tilting field is decoupled into normalized stretching $\mathbf{S}$ and normalized tilting $\mathbf{T}$ following Violato and Scarano, ${ }^{19}$ which are plotted in Figure 9 as color code of $\lambda_{2} D / W_{j}$ iso-surface. Peak activity of absolute stretching $|\mathbf{S}|$ and absolute tilting $|\mathbf{T}|$ is observed when the periphery of the toroidal vortex disrupts into "C" structures and the inner portions of the vortex "P" are ejected. Stretching and tilting fields show similar peak magnitude, $|\mathbf{S}|=3$ and $|\mathbf{T}|=3$, which is comparable to the stretching and tilting activity of streamwise vortices (Violato and Scarano ${ }^{19}$ ). Beyond the end of the potential core, $|\mathbf{S}|$ and $|\mathbf{T}|$ peak activities decrease to 1 in correspondence to the "VF" structures as well as in the region closer to the jet axis where the coherence decays faster.

A statistical characterization of the stretching and tilting activity along the jet axis is shown in Figure 10, where absolute stretching $|\mathbf{S}|$ and absolute tilting $|\mathbf{T}|$ fields are averaged in time and along the azimuthal direction between $R / D=0$ and $R / D=1,\langle|\mathbf{S}|\rangle$ and $\langle|\mathbf{T}|\rangle$, respectively. Between $Z / D=5.5$ and 6 , where toroidal vortices undergo breakdown, $\langle|\mathbf{S}|\rangle$ and $\langle|\mathbf{T}|\rangle$ reach peak activity, although with maximum tilting $(\langle|\mathbf{T}|\rangle=0.72$ at $\mathrm{Z} / \mathrm{D}=5.5)$ that is $20 \%$ larger than the peak of stretching. Further downstream (Z/D > 6.5), in correspondence with the formation of "VF" structures and the coherence decay, $\langle|\mathbf{S}|\rangle$ and $\langle|\mathbf{T}|\rangle$ decrease to values that are comparable to those found for vortex pairing and early growth of azimuthal and streamwise vortices $(3.5<\mathrm{Z} / \mathrm{D}<4$, Violato and Scarano ${ }^{19}$ ).

\section{Acoustic source characterization of jet core breakdown}

The relation between the large-scales structures and the local activity of acoustic sources in the region across the end of the potential core is explored recalling Powell's aeroacoustic analogy (1964). The acoustic source can be mapped using the norm of the second time derivative of the Lamb 


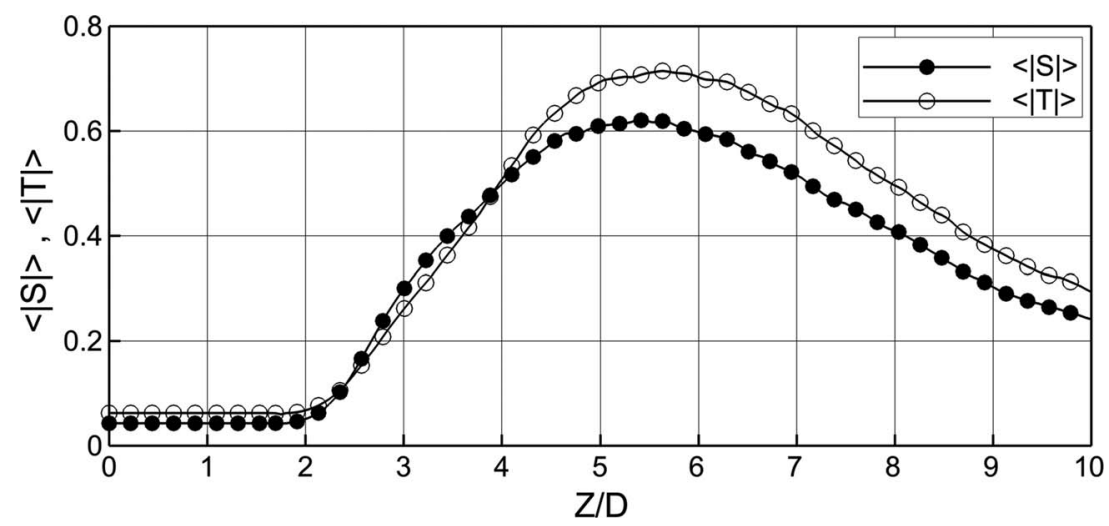

FIG. 10. Axial distribution of space-time average of $|\mathrm{S}|$ and $|\mathrm{T}|$.

vector $\left(\ddot{L}=\left|\left(d^{2} \mathbf{L} / d t^{2}\right)\right| D^{3} / W_{j}^{4}\right)$. The spatio-temporal patterns of $\ddot{L}$ are discussed in relation with the toroidal vortex breakdown and the formation of vortex filaments beyond the end of the potential core.

The temporal sequence of Figure 11, illustrates the vortex patterns evolution $\left(\lambda_{2} D / W_{j}\right.$ isosurfaces) and the corresponding acoustic source field (iso-surfaces of $\ddot{\mathrm{L}}$ ) during the breakdown process of a toroidal structure. Before breakdown $\left(t W_{j} / D=0\right)$, the acoustic source shows a strongly distorted toroidal configuration corresponding to the vortex ring. Subsequently, during the loss of azimuthal coherence $\left(0.84<t W_{j} / D<1.68\right)$, the acoustic source becomes more pronounced in the region closer to the jet axis, where portions " $\mathrm{P}$ " of the vortex ring (Figure 6) are tilted and ejected along the jet axis. On the other hand, the rupture of the vortex into " $\mathrm{C}$ " structures is not associated to significant activity in terms of $\ddot{\mathrm{L}}$.

The pattern shown in Figure 12 yields evidence that the activity of $\ddot{\mathrm{L}}$ is more intense for core breakdown than for the case of vortex pairing (Hussain and $\mathrm{Zaman}^{2}$ ). The transient phenomenon
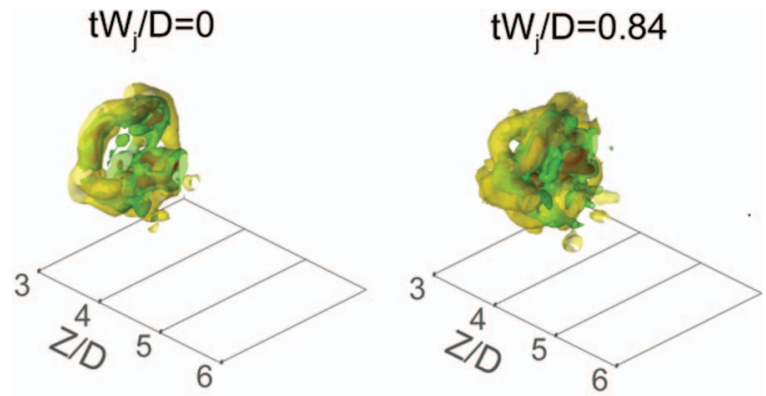

$$
t W_{j} / D=1.68
$$

$\mathrm{tW}_{\mathrm{j}} / \mathrm{D}=2.52$
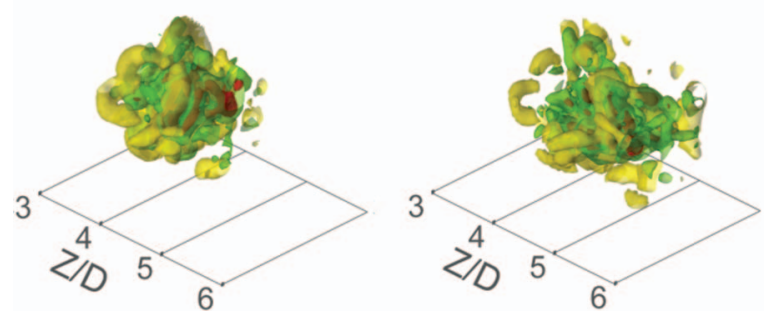

FIG. 11. Time sequence visualization of vortex ring breakdown (iso-surfaces $\lambda_{2} D / W_{j}=-0.8$ in yellow) with acoustic source (iso-surfaces $\ddot{\mathrm{L}}=30$ in green and $\ddot{\mathrm{L}}=50$ in red). 


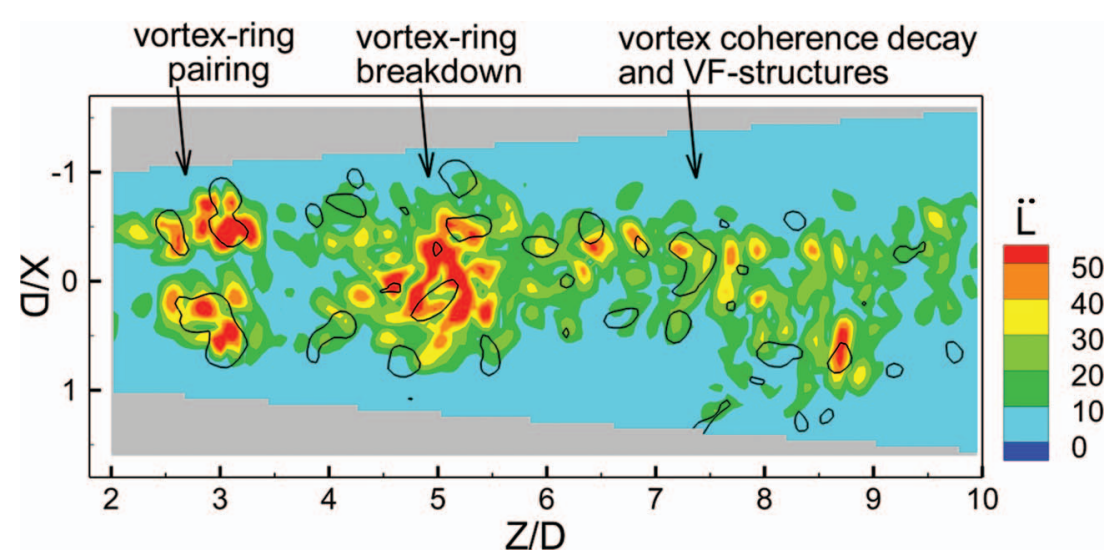

FIG. 12. Instantaneous iso-contour plot of $\ddot{\mathrm{L}}$ and iso-line $\lambda_{2} D / W_{j}=-0.8$ identifying coherent structures at $t W_{j} / D=1.68$.

of core breakdown, however, weakens rapidly and, beyond the end of the potential core, the region interested by $\ddot{\mathrm{L}}$ activity collapses within 3 jet diameters. The peripheral region $(0.8<R / D<1.6)$ exhibits "VF" structures (Figure 7) but without sound production activity.

The axial distribution of the source is obtained performing an ensemble average of $\ddot{\mathrm{L}}$ over the observation time and considering homogeneous properties in the azimuthal direction $(\overline{\mid \ddot{\mathbf{L}}})$. The result is shown in Figure 13, where $\overline{\langle\ddot{\mathrm{L}}\rangle}$ increases rapidly between 2 and 3 diameters due to the vortex pairing. A subsequent increase of comparable magnitude yields peak activity of $\overline{\langle\ddot{\mathrm{L}}\rangle}$ between $Z / D$ $=5.5$ and 6 where the portions "P" of vortex rings are stretched, tilted and then axially ejected yielding the disruption of the toroidal coherence (Figure 6). Beyond the end of the potential core, $\overline{\langle\ddot{\mathrm{L}}\rangle}$ eventually decays and at 10 jet diameters the value is lower than that in the pairing region.

\section{3D POD analysis}

In this section, snapshot-POD (Sirovich ${ }^{38}$ ) is applied to inspect the large-scale features developing in the jet with attention to the three-dimensional regime. The analysis is conducted on fields of velocity $(\mathbf{V})$, vorticity $(\boldsymbol{\omega})$, and Lamb vector $(\mathbf{L})$ fields as well as second time derivative of the Lamb vector $\left(d^{2} \mathbf{L} / d t^{2}\right)$, which identifies the acoustic source. The decomposition of velocity, vorticity, and Lamb vector fields is based on a sequence of 500 uncorrelated snapshots, which corresponds to an observation length of 1000 jet diameters. The POD analysis of $d^{2} \mathbf{L} / d t^{2}$ fields, instead, is conducted on a subset of snapshots that are extracted with a sampling rate of $100 \mathrm{~Hz}$ from the time-resolved

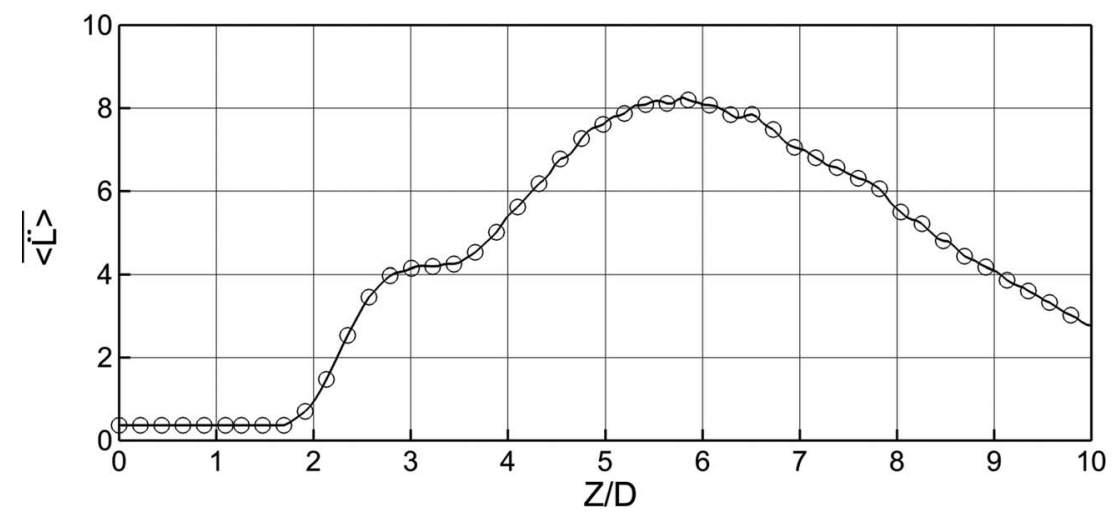

FIG. 13. Space-time average of $\ddot{\mathrm{L}}$. 

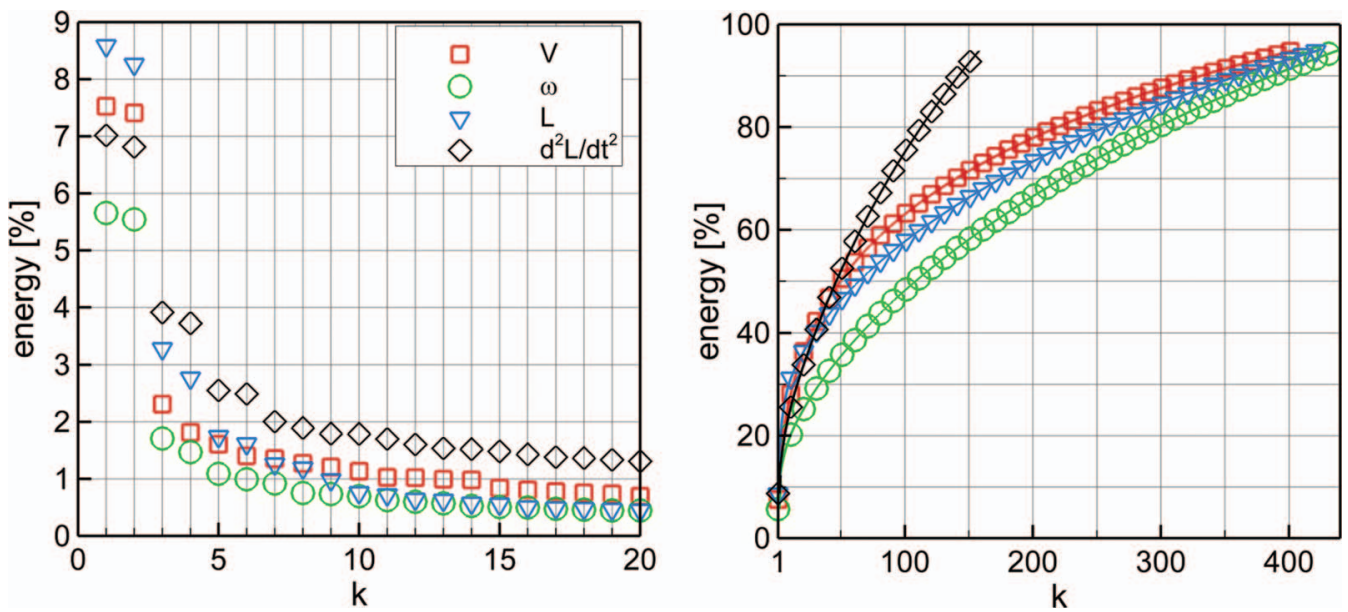

FIG. 14. Left: energy distributions across first 20 modes of velocity $\mathbf{V}$, vorticity $\boldsymbol{\omega}$, Lamb vector $\mathbf{L}$ and second time derivative of the Lamb vector $d^{2} \mathbf{L} / d t^{2}$; right: cumulative energy distributions (symbols are shown 1 every 10). Note that the observation length employed for $d^{2} \mathbf{L} / d t^{2}$ decomposition is shorter compared to $\mathbf{V}, \boldsymbol{\omega}$ and $\mathbf{L}$.

sequence. A total of 190 snapshots are employed, which correspond to an observation length of 100 jet diameters that is shorter compared to the velocity, vorticity, and Lamb vector ones.

The distributions of energy across the three-dimensional POD modes of velocity, vorticity, Lamb vector, and its second time derivative fields are illustrated in Figure 14 together with the cumulative energy distributions. Mode 1 and 2 of the velocity correspond to $7.5 \%$ and $7.4 \%$ of the total kinetic energy (Eq. (10)), whereas less enstrophy (Eq. (11)) corresponds to vorticity mode 1 and $2(5.7 \%$ and 5.6\%). The Lamb vector is decomposed with a slightly higher efficiency, where mode 1 and 2, respectively, capture $8.6 \%$ and $8.3 \%$ of the total Lamb energy (Eq. (12)). Instead, the first pair of modes of the $d^{2} \mathbf{L} / d t^{2}$ decomposition are, respectively, associated with $7.01 \%$ and $6.81 \%$ of the total acoustic source energy (Eq. (13)).

The spatial organization of the first 10 dominant POD modes of velocity (Figures 15 and 16), vorticity (Figures 17 and 18), Lamb vector (Figures 19 and 20), and Lamb vector second time derivative (Figure 21) are described and the related information on the characteristic frequencies are extracted from the spectrum of the time coefficients $a_{k}(t)$ (Eq. (5)) of the modes. The peak frequencies of velocity, vorticity, Lamb vector, and Lamb vector second time derivative are summarized in Figure 23.

\section{Decomposition based on velocity}

The POD method is now applied to the 3D velocity fields. Figure 14 shows that about $50 \%$ of the kinetic energy is captured by the first 50 modes, which is far less than the 350 modes reported by Jordan $e t a l .{ }^{5}$ for a compressible jet at $R e=3600$. In the first 10 velocity modes, which corresponds to $27.5 \%$ of the kinetic energy, there are pairs (1 and 2, 4 and 5, 6 and 7, 9 and 10) phase shifted of $\pi / 2$ in the axial direction describing travelling waves (Figure 15). Non-travelling (presumably pulsatile) modes ( 3 and 8 ) are instead illustrated in Figure 16. For conciseness, Figure 15 shows the iso-surfaces of $W / W_{j}, V_{r} / W_{j}$, and $V_{\theta} / W_{j}$ of only one mode for each pair. Mode 1 and 2 describe travelling toroidal vortices (Bi et al. ${ }^{47}$ Shinneeb et al. ${ }^{48}$ Jung et al.,${ }^{11}$ and Lynch and Thurow ${ }^{13}$ ) that are formed after pairing and have characteristic Strouhal number $S t=0.36$ (Figure 23).

The axial velocity component shows a pulsatile motion along the axial direction, whereas the azimuthal velocity reveals the presence of "periodic" twisting that develop in the inner region of the vortices. In mode no. 4 and 5 , across the end of the potential core $(6<Z / D<8)$, the intertwining between the region of positive and negative $W / W_{j}$ indicates a helical motion. This was previously reported by Iqbal and Thomas ${ }^{12}$ who observed it beyond the end of the potential core. A similar observation was made by Lynch and Thurow. ${ }^{13}$ Further downstream $(\mathrm{Z}>8)$, instead, $W / W_{j}$ describe a flapping motion (Lynch and Thurow ${ }^{13}$ ). The aforementioned motions are coupled with a wave-like 

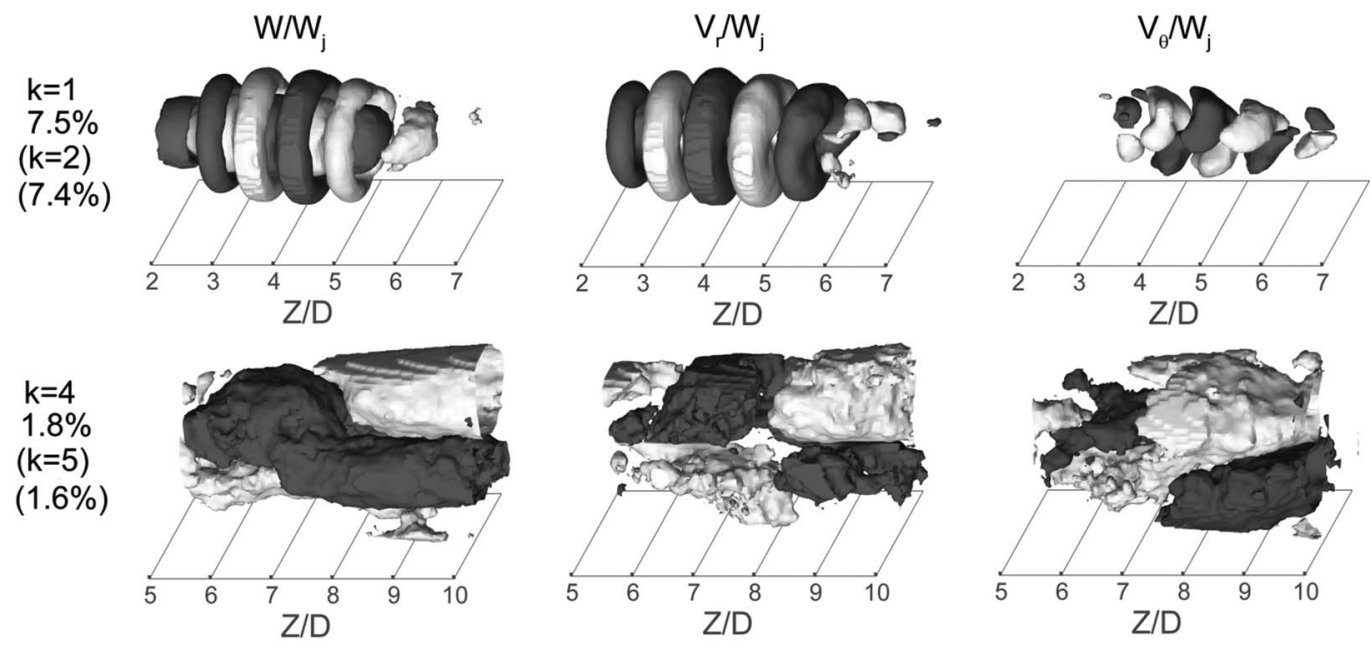

$\mathrm{k}=6$

$1.4 \%$

$(\mathrm{k}=7)$

$(1.35 \%)$
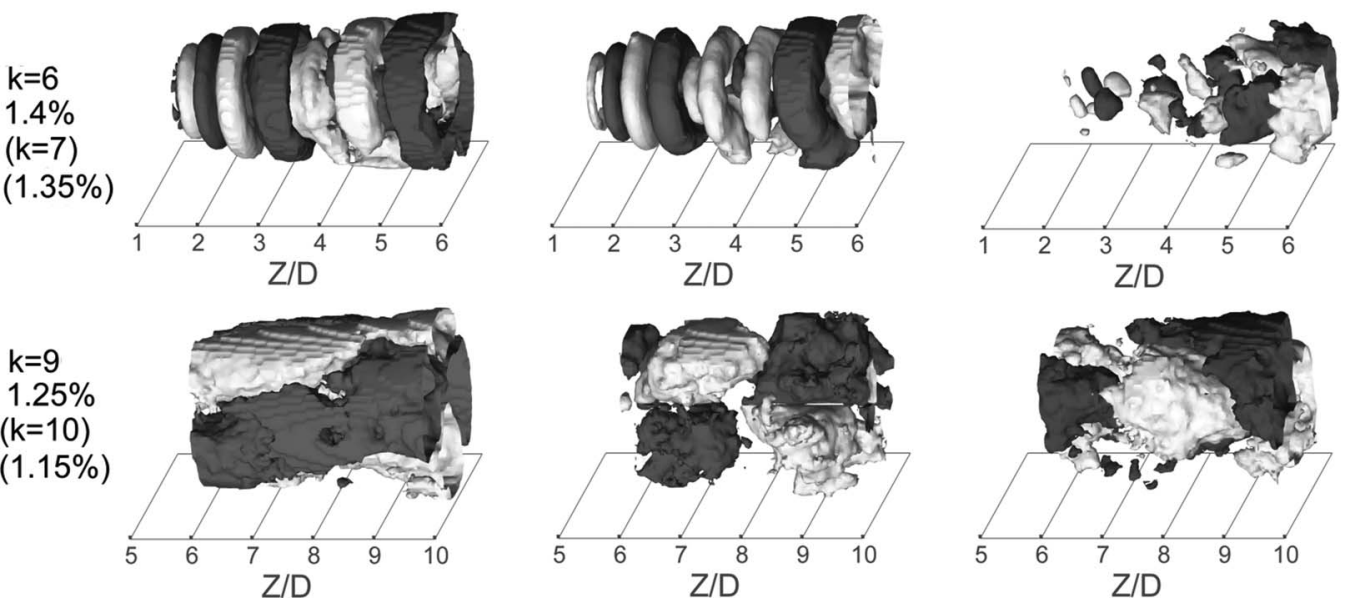

FIG. 15. POD modes of velocity describing travelling waves. Between brackets mode number and energy of the coupled mode. Positive (light grey) and negative (dark grey) iso-surfaces of $W / W_{j}$ (left), $V_{r} / W_{j}$ (center), and $V_{\theta} / W_{j}$ (right).
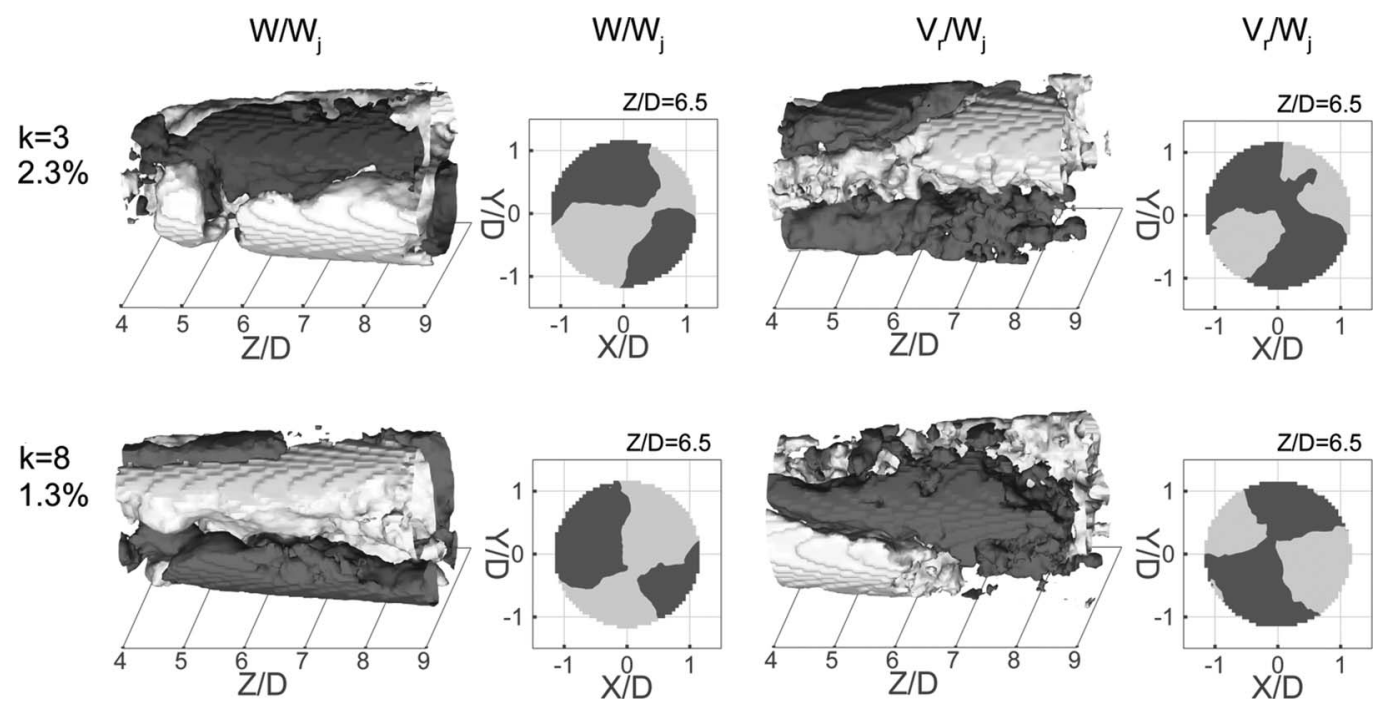

FIG. 16. POD modes of velocity describing pulsatile motions. Positive (light grey) and negative (dark grey) iso-surfaces of $W / W_{j}$ (left), $V_{r} / W_{j}$ (right), and cross-sectional iso-contours. 

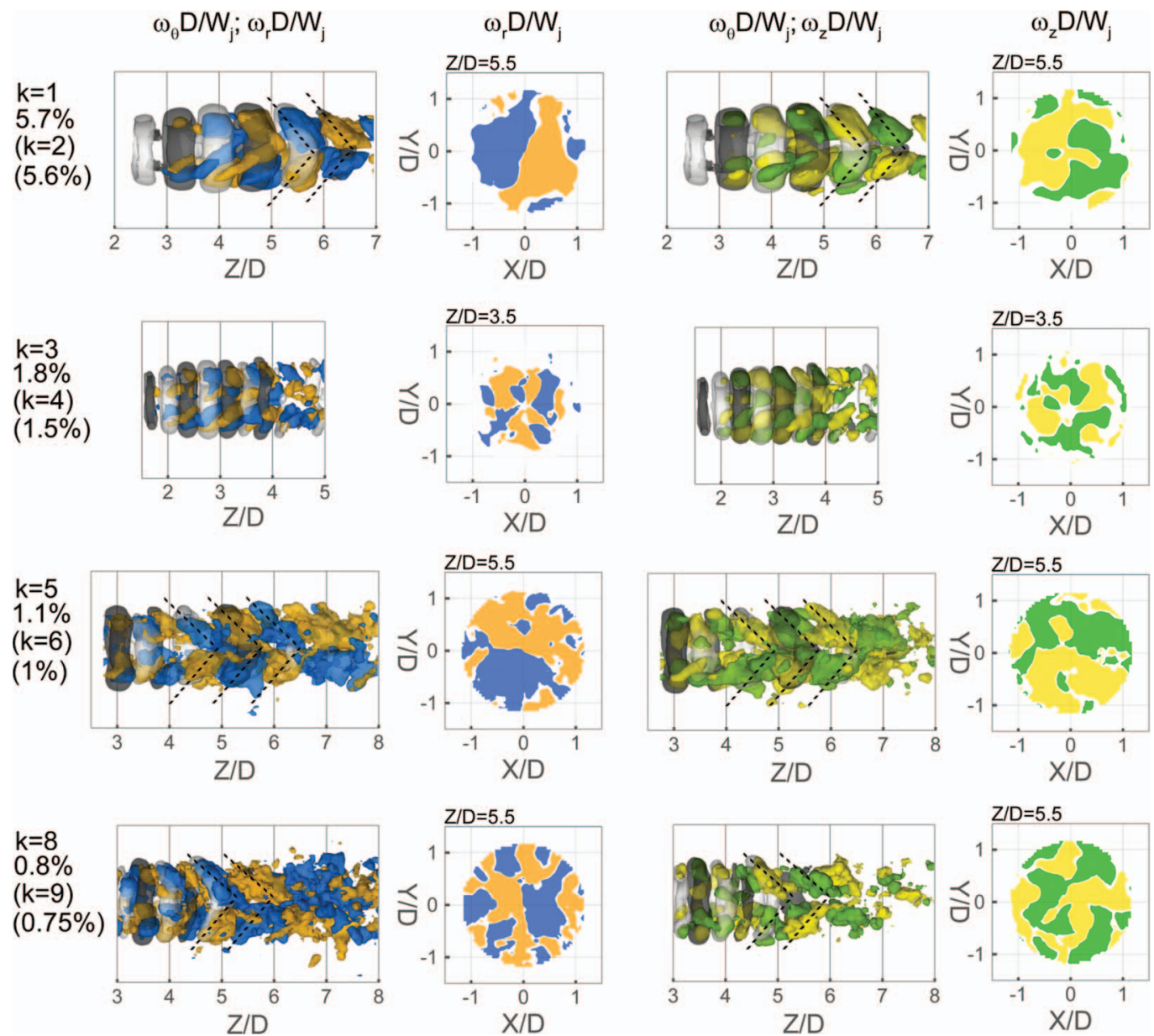

FIG. 17. POD modes of vorticity describing travelling waves. Between brackets mode number and energy of the coupled mode. Left: iso-surfaces of $\omega_{\theta} D / W_{j}$ (positive in light grey; negative in dark grey) and $\omega_{r} D / W_{j}$ (positive in orange and negative in blue), and cross-sectional iso-contour of $\omega_{r} D / W_{j}$. Right: Iso-surfaces of $\omega_{\theta} D / W_{j}$ (positive in light grey; negative in dark grey) and $\omega_{z} D / W_{j}$ (positive in yellow and negative in green) and cross-sectional iso-contour of $\omega_{z} D / W_{j}$ with iso-line $\omega_{\theta} D / W_{j}$.

$$
\omega_{\theta} \mathrm{D} / \mathrm{W}_{\mathrm{j}} ; \omega_{\mathrm{r}} \mathrm{D} / \mathrm{W}_{\mathrm{j}}
$$

$\mathrm{k}=7$

$0.95 \%$
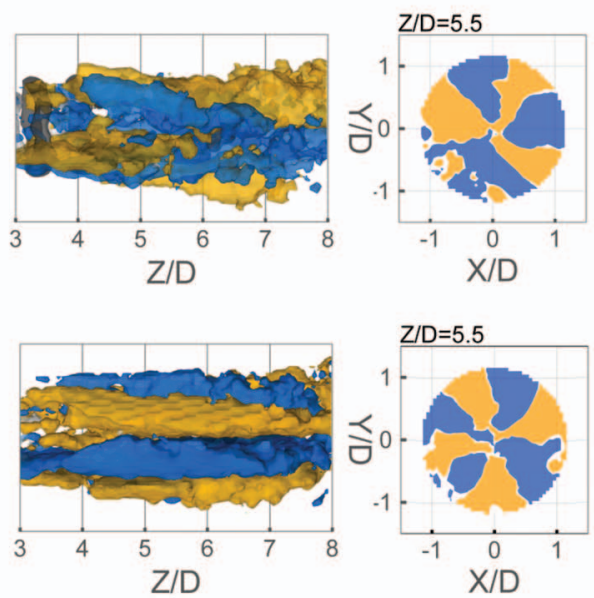

$\omega_{\theta} \mathrm{D} / \mathrm{W}_{\mathrm{j}} ; \omega_{\mathrm{z}} \mathrm{D} / \mathrm{W}_{\mathrm{j}}$
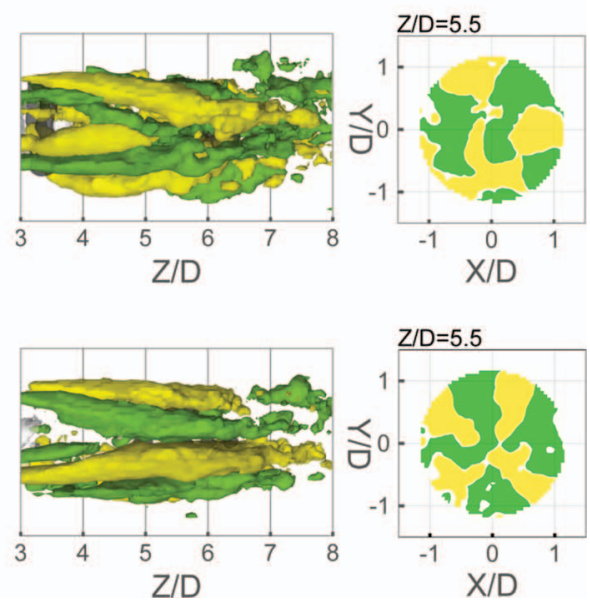

FIG. 18. POD modes of vorticity describing pulsatile motions (refer to caption of Figure 17 for the legend). 

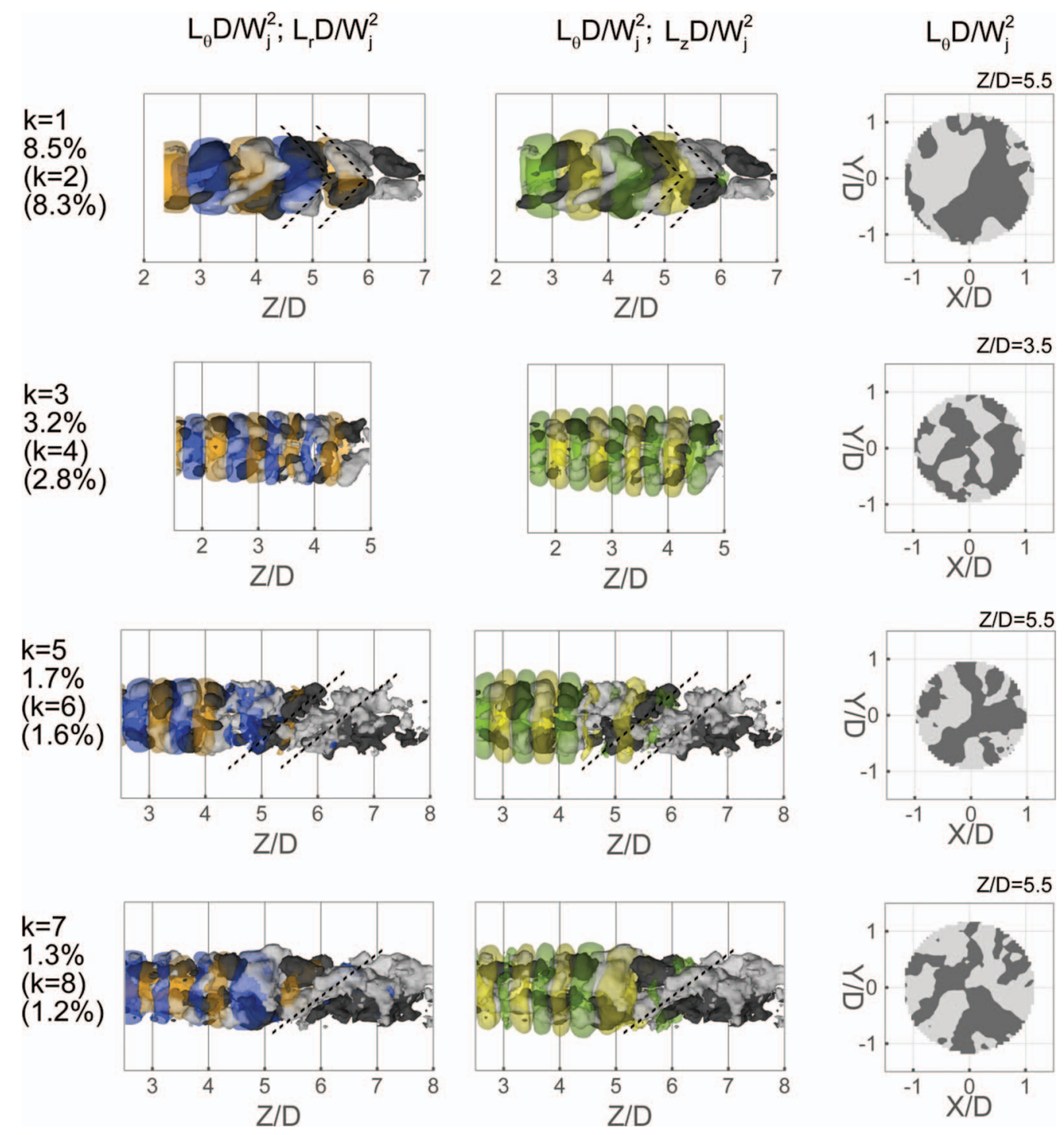

FIG. 19. POD modes of Lamb vector describing travelling waves. Between brackets mode number and energy of the coupled mode. Left: iso-surfaces of $L_{\theta} D / W_{j}$ (positive in light grey; negative in dark grey) and $L_{r} D / W_{j}$ (positive in orange and negative in blue); centre: iso-surfaces of $L_{\theta} D / W_{j}$ (positive in light grey; negative in dark grey) and $L_{z} D / W_{j}$ (positive in yellow and negative in green); right: cross-sectional iso-contour of $L_{\theta} D / W_{j}$.

motion $\left(V_{r} / W_{j}\right)$ and a periodic twisting $\left(V_{\theta} / W_{j}\right)$ that have 4 jet diameters of spatial wavelength. Mode 4 and 5 are associated with a primary frequency $S t=0.3$ and a secondary of 0.7. Mode 6 and 7 describe travelling toroidal vortices after shedding, which are periodically twisted between $Z / D=3$ and $Z / D=6$ with a wavelength of 1 jet diameter $(S t=0.72)$. Mode 9 and 10 show two filaments of positive and two filaments of negative $W / W_{j}$, that are phased shifted of $\pi / 2$ on the plane normal to the jet axis and develop downstream the end of the potential core. They describe a precession motion. This is coupled with a wave-like motion $\left(V_{r} / W_{j}\right)$ and a periodic twisting $\left(V_{\theta} / W_{j}\right)$ that are similar to those observed in mode 4 and 5. Primary and secondary Strouhal peaks of pair 9-10 are 0.36 and 0.7 Modes 3 and 8 are characterized by the axial development of four filaments of $W / W_{j}$ and $V_{r} / W_{j}$, two of positive sign and two of negative sign. They describe axial and radial pulsatile motions $(4<Z / D<9)$ with a phase shift of about $45^{\circ}$ in the azimuthal direction (Figure 16) and a discrepancy in the associated energy of about $1 \%$. Moreover, while they are characterized by similar secondary Strouhal peak (0.7), they differ for the primary one. 

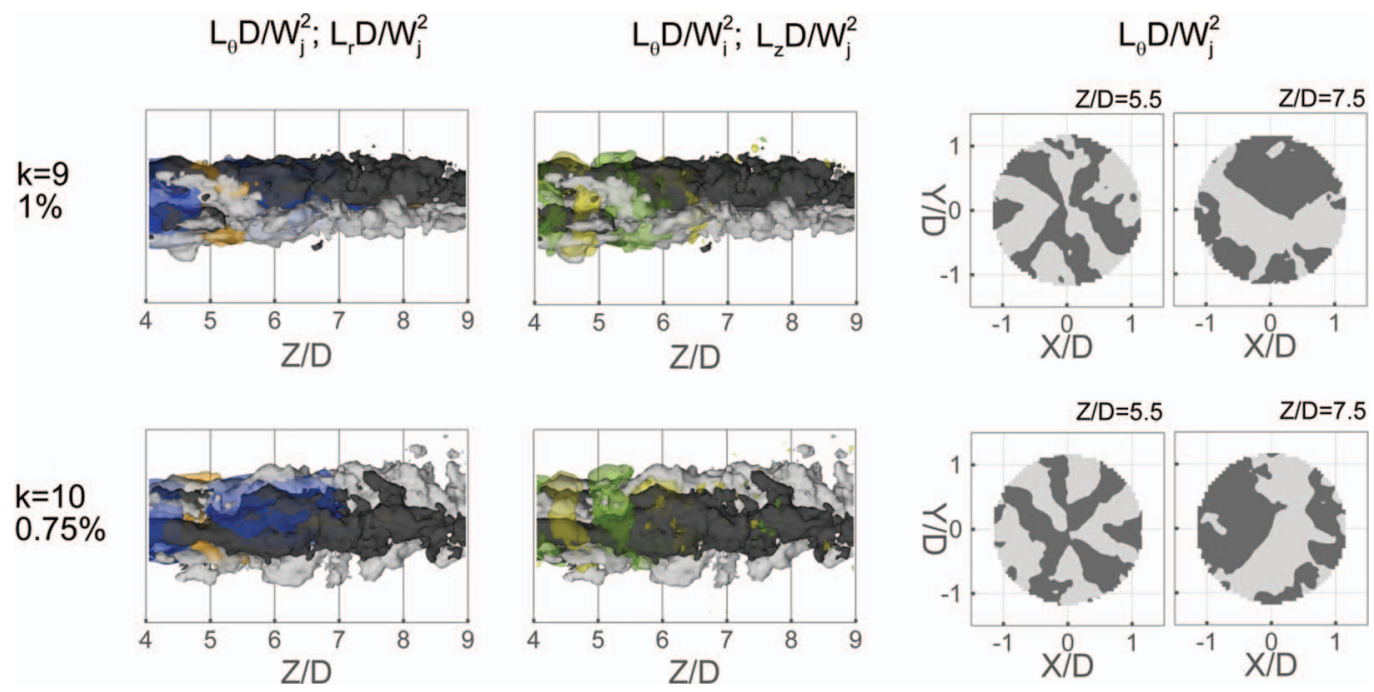

FIG. 20. POD modes of Lamb vector describing pulsatile motions (refer to caption of Figure 19 for the legend).

\section{Decomposition based on vorticity}

The POD method applied to the vorticity fields highlights the dynamical organization of coherent vortices. In the first 10 vorticity modes, which correspond to $20 \%$ of the flow enstrophy, there are pairs ( 1 and 2, 3 and 4, 5 and $6, \# 8$ and \#9) phase shifted of $\pi / 2$ in the axial direction describing travelling waves. For conciseness, only one mode for each pair is reported in Figure 17. Pulsatile modes (7 and 10) are instead illustrated in Figure 18.

Travelling ring vortices after pairing are described by modes 1 and 2, which are the most energetic (5.7\% and 5.6\% of the total energy), as found from the velocity decomposition. The same type of motion is also associated with modes 5 and 6 with lower energy content. The threedimensional pattern that evolves in conjunction travelling vortex rings and that, ultimately, leads to the breakdown, is illustrated by iso-surfaces of radial and axial components of the vorticity modes, $\omega_{r} D / W_{j}$ and $\omega_{z} D / W_{j}$ as well as by cross-sectional iso-contours plots of the same quantities. Characterized by peak Strouhal $S t=0.36$ (Figure 23), modes 1 and 2 show that the motion of vortex rings after pairing (see isosurfaces of $\omega_{\theta} D / W_{j}$ ) is accompanied by travelling waves of $\omega_{r} D / W_{j}$ and $\omega_{z} D / W_{j}$ that develop across the end of the potential core. For $Z / D>4.5$, they show a characteristic inclination of $40^{\circ}-45^{\circ}$ to the jet axis (see dashed lines). Phase opposition on the plane normal to the jet axis (see iso-contour plots at $Z / D=5.5$ ) is observed for $\omega_{r} D / W_{j}$ and $\omega_{z} D / W_{j}$, indicating that while one half of the ring structure is subjected to inward radial swirl and positive axial swirl, the other half undergo outward radial swirl and negative axial swirl. Modes 3 and 4 describe travelling ring vortices after shedding (see iso-surface of $\omega_{\theta} D / W_{j}, S t=0.72$ ) with spatial wavelength of 0.75 , half that of pairing mode (modes 1 and 2), in the region between $Z / D=1.5$ and 4 . The motion is combined with travelling waves of $\omega_{r} D / W_{j}$ and $\omega_{\theta} D / W_{j}$ that, as illustrated in the iso-contour plot at $Z / D=3.5$, are distributed along the azimuthal direction with wave number $k=4$, meaning of four pairs of counter-rotating streamwise vortices, which agrees with previous experiments (Violato and Scarano ${ }^{19}$ ). Similarly to 1 and 2, modes 5 and 6 describe the motion of vortex rings after pairing together with travelling waves of $\omega_{r} D / W_{j}$ and $\omega_{z} D / W_{j}$ with a characteristic inclination of $40^{\circ}-45^{\circ}$ to the jet axis in the region $4<Z / D<6.5$. However, compared to modes 1 and 2 , they are rotated of $\pi / 2$ around the jet axis and they also feature secondary Strouhal peak $S t=0.7$. More three-dimensional features are identified by the pair of modes 8 and 9 which describe travelling "wave" of radial and axial vorticity filaments that also have a preferred orientation at $45^{\circ}$ to the jet axis in the region 4.5 $<\mathrm{Z} / \mathrm{D}<6$ (related Strouhal peaks $S t=0.31$ and 0.7 ). 

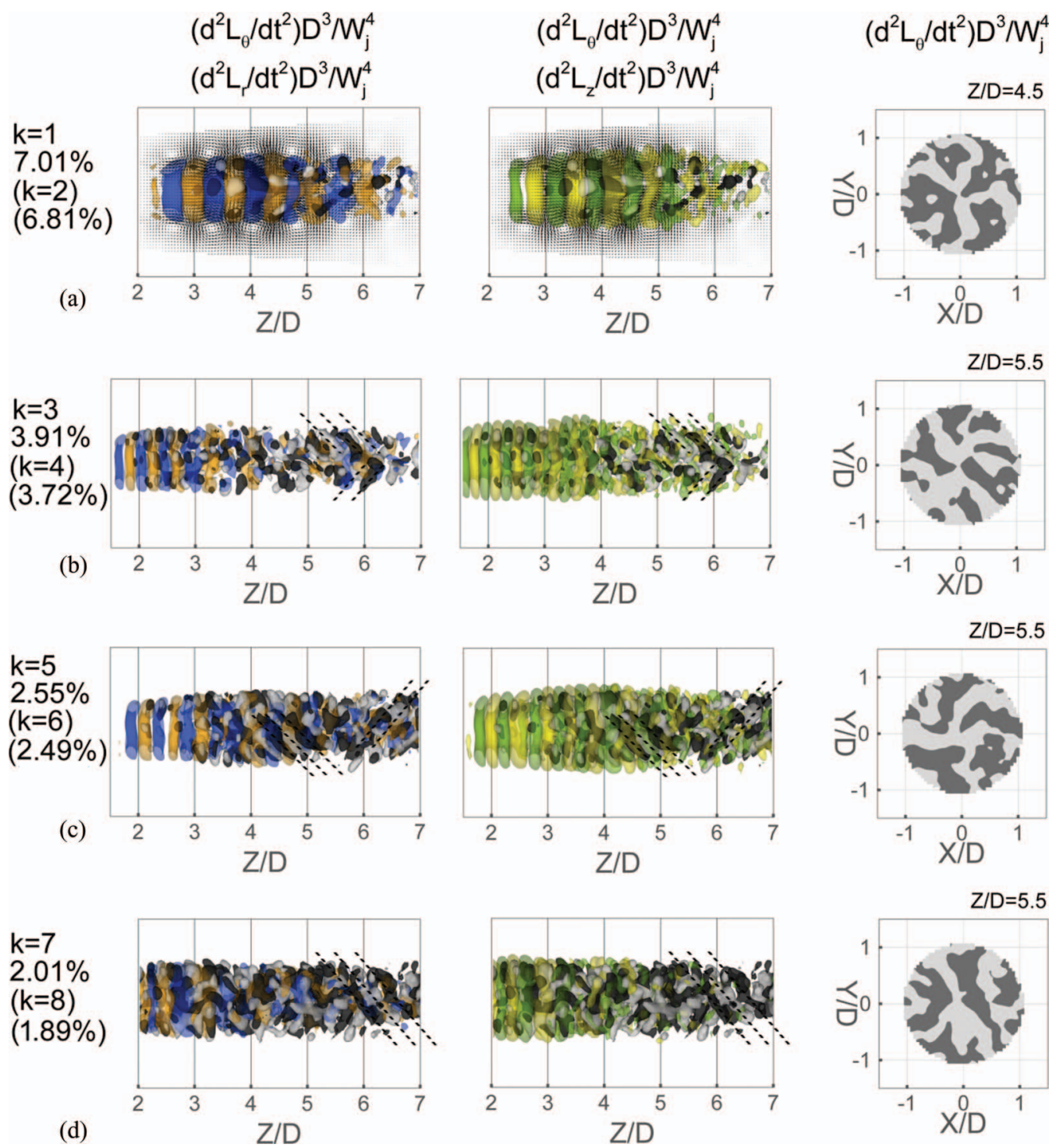

FIG. 21. POD modes of the second time derivative of the Lamb vector describing travelling waves. Between brackets mode number and energy of the coupled mode. Left: iso-surfaces of $\left(d^{2} L_{\theta} / d t^{2}\right) D^{3} / W_{j}^{4}$ (positive in light grey; negative in dark grey) and $\left(d^{2} L_{r} / d t^{2}\right) D^{3} / W_{j}^{4}$ (positive in orange and negative in blue); centre: iso-surfaces of $\left(d^{2} L_{\theta} / d t^{2}\right) D^{3} / W_{j}^{4}$ (positive in light grey; negative in dark grey) and $\left(d^{2} L_{z} / d t^{2}\right) D^{3} / W_{j}^{4}$ (positive in yellow and negative in green); right: cross-sectional iso-contour of $\left(d^{2} L_{\theta} / d t^{2}\right) D^{3} / W_{j}^{4}$.

The characteristic $45^{\circ}$ inclination of the radial and the axial vorticity that is observed in mode pairs 1-2, 5-6, and 8-9 is associated with the process of vortex ring breakdown by axial ejection of vortex humps (Figure 6).

Pulsatile modes 7 and 10 (Figure 18) show four pairs of counter-rotating streamwise filaments of $\omega_{r} D / W_{j}$ and $\omega_{z} D / W_{j}$ that develop across the region of vortex breakdown between $\mathrm{Z} / \mathrm{D}=3.5$ and $\mathrm{Z} / \mathrm{D}=8$ and are, respectively, associated with Strouhal peak $S t=0.79$ and $S t=0.15$. These structures may be associated to the propeller-like vortex pattern already reported by Jung et al. ${ }^{11}$

\section{Decomposition based on Lamb vector}

The POD analysis of the Lamb vector, which in Powell's aeroacoustic analogy accounts for the flow state (see the Appendix), shows the first 10 modes capturing $30.3 \%$ of the total Lamb 
energy. Travelling waves are described by the first 4 pairs of modes ( 1 and 2, 3 and 4, 5 and 6 , 7 and 8), illustrated in Figure 19. While in the vorticity modes toroidal vortices are described by the azimuthal vorticity component (Figure 17), in the Lamb vector modes the azimuthal coherence is described by the radial and the axial components $L_{r} D / W_{j}$ and $L_{z} D / W_{j}$. The development of a three-dimensional disturbance is instead described by the azimuthal component $L_{\theta} D / W_{j}$ (Figures 19 and 20). Similarly to the velocity and vorticity decompositions, the first and the second mode of the Lamb vector describe the motion of toroidal vortices formed after pairing $(S t=0.36$, Figure 23) and the presence of a three-dimensional disturbance (see isosurface $L_{\theta} D / W_{j}$ ) that develop across the end of the potential core. This disturbance grows with a characteristic $40^{\circ}-45^{\circ}$ inclination to the jet axis, similarly to the waves $\omega_{r} D / W_{j}$ and $\omega_{z} D / W_{j}$ observed in pairs 1-2, 5-6, and 8-9 of the vorticity decomposition. In the region between $Z / D=2.5$ and 5, modes 3-4 describes travelling toroidal vortices after shedding $(S t=0.72)$, as observed for the vorticity decomposition. The motion is combined with travelling wave of $L_{\theta} D / W_{j}$ that show an azimuthal distribution with a wave number $k$ $=4$, as observed in mode 3-4 for the radial and axial component of the vorticity (see cross-sectional plots at $Z / D=3.5$ ). Modes 5-6, as well as modes 7-8, describe the motion of vortices after shedding in the region between $Z / D=2.5$ and 5 (note that the related Strouhal is $S t=0.7$, slightly slower than that of mode 3-4). Further downstream, in the region across the end of the potential core $(5<Z / D<7)$, filaments of $L_{\theta} D / W_{j}$ (see cross-sectional plots at $\mathrm{Z} / \mathrm{D}=5.5$ ) identify a travelling wave characterized by axial swirling with an angle of $40^{\circ}-45^{\circ}$ to the jet axis. Such motion is associated with $S t=0.93$ for mode \#5-\#6 and $S t=0.6$ for mode \#7-\#8.

Lamb vector pulsatile modes 9 and 10 (Figure 20) show 6 main pairs of streamwise filaments of $L_{\theta} D / W_{j}$ coherence that develop until the region of vortex breakdown $(Z / D=5.5)$ and then merge into a main pair $(Z / D=7.5)$. As shown in the cross-sectional contour plots of $L_{\theta} D / W_{j}$ at $Z / D=7.5$ (Figure 20), mode 10 is rotated of about $\pi / 2$ around the jet axis with respect to mode 9 . The related Strouhal peak is 0.64 for both modes.

\section{Decomposition based on Lamb vector second time derivative}

The first 10 POD modes of the Lamb vector second time derivative $\left(d^{2} \mathbf{L} / d t^{2}\right)$ capture $31.9 \%$ of the total acoustic source energy (Eq. (13)), as shown in Figure 14. The first 5 pairs of modes ( 1 and 2, 3 and 4,5 and 6,7 and 8,9 and 10) identify travelling waves as shown in Figure 21. As seen in the Lamb vector modes, the azimuthal coherence is identified by the radial and the axial components $\left(\left(d^{2} L_{r} / d t^{2}\right) D^{3} / W_{j}^{4}\right.$ and $\left.\left(d^{2} L_{z} / d t^{2}\right) D^{3} / W_{j}^{4}\right)$ which, in the region upstream of the end of the potential core, resemble a wave-packet organization (Cavalieri et al. ${ }^{49,50}$ ). On the other hand, the azimuthal component $\left(d^{2} L_{\theta} / d t^{2}\right) D^{3} / W_{j}^{4}$ typically identifies the development of three-dimensional disturbances.

The first pair of $d^{2} \mathbf{L} / d t^{2}$ modes describes the axisymmetric motion related to toroidal vortices after pairing $\left(S t=0.36\right.$, Figure 23). The vortices identified by $\left(d^{2} L_{r} / d t^{2}\right) D^{3} / W_{j}^{4}$ and $\left(d^{2} L_{z} / d t^{2}\right) D^{3} / W_{j}^{4}$ have spatial wavelength that is half of the pairing modes, as illustrated in Figure 21 where the iso-surfaces of mode $k=1$ are plotted together with the midplane vector field of the velocity mode 1 . On the other hand, by the end of the potential core $(4.5<Z / D<6.5)$, where vortex rings disrupt (Figure 6) and the acoustic source is more pronounced (Figures 11 and 13), the iso-surfaces of $\left(d^{2} L_{r} / d t^{2}\right) D^{3} / W_{j}^{4}$ and $\left(d^{2} L_{z} / d t^{2}\right) D^{3} / W_{j}^{4}$ show a gradual decay of the axisymmetric coherence. By colour-coding these iso-surfaces with iso-contour of $Z / D$ (Figure 22), it is possible to observe four humps ("Ps"), meaning of azimuthal instabilities with a wave number $k=4$. This wave number is also reported for the radial and the axial components of vorticity modes 3-4 (Figure 17) and 10 (Figure 18), as well as for the azimuthal component of Lamb vector modes 3-4 (Figure 19).

While in modes 1-2 the iso-surfaces $\left(d^{2} L_{\theta} / d t^{2}\right) D^{3} / W_{j}^{4}$ do not exhibit any recurrent pattern, in modes 3-4 they identify filaments at $40^{\circ}-45^{\circ}$ to the jet axis, as similarly reported for the vorticity and the Lamb vector modes (Figures 17 and 19). Such filaments describe travelling waves in the region by the end of the potential core $(4.5<Z / D<6)$, where vortex rings disrupt (Figure 6$)$ and the acoustic source becomes pronounced (Figures 11 and 13). On the other hand, the radial and the axial components of modes 3-4 describe travelling toroidal vortices that are related to the vortex 

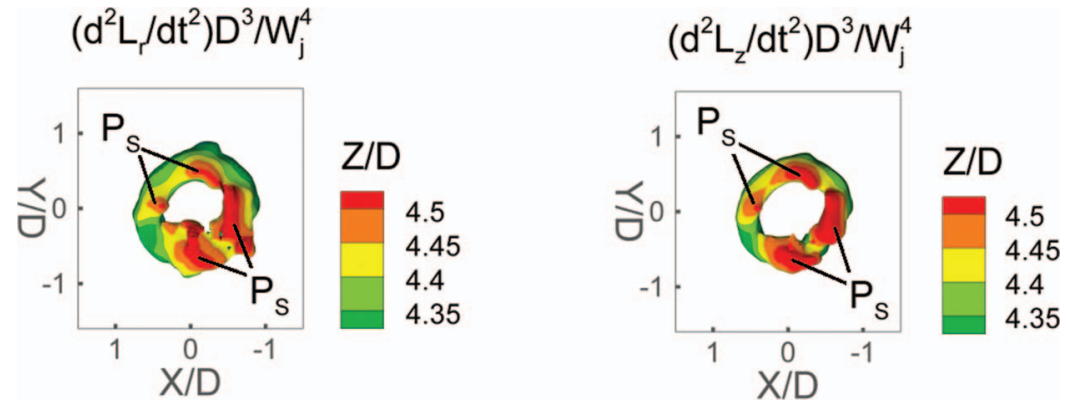

FIG. 22. Detail of mode 1 of the second time derivative of the Lamb vector between $Z / D=4.3$ and 4.5. Positive iso-surfaces of $\left(d^{2} L_{r} / d t^{2}\right) D^{3} / W_{j}^{4}$ and $\left(d^{2} L_{z} / d t^{2}\right) D^{3} / W_{j}^{4}$ color coded with $Z / D$.

shedding. Note that the spatial wavelength shown is half of the shedding modes observed in the velocity, vorticity, and Lamb vector decomposition. Modes 3-4 are associated with the shedding frequency $(S t=0.72)$ of ring vortices, and with a secondary frequency $(S t=1.9)$, that can instead be attributed to the three-dimensional travelling wave in the region by the end of the potential core.

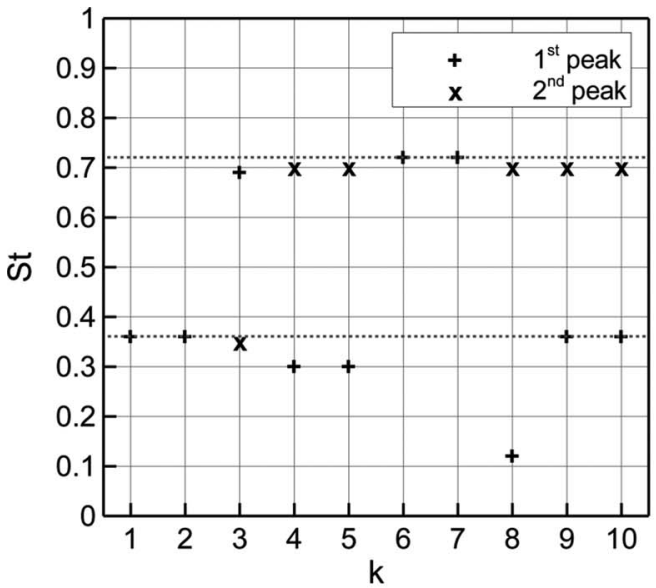

(a)

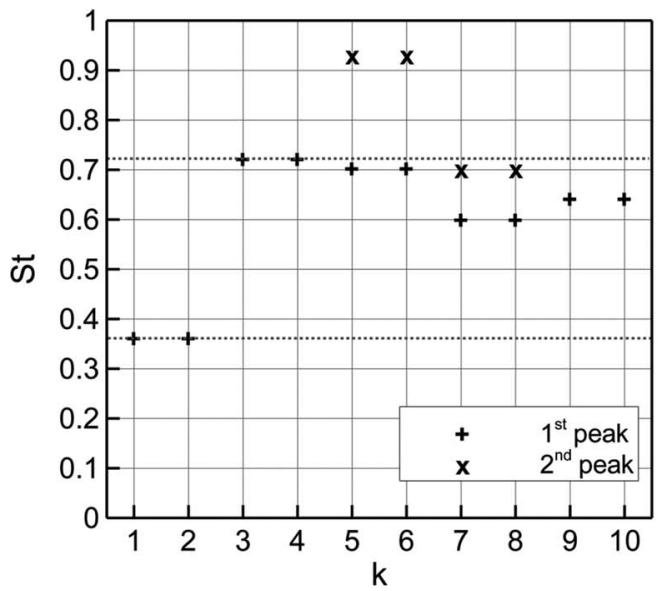

(c)

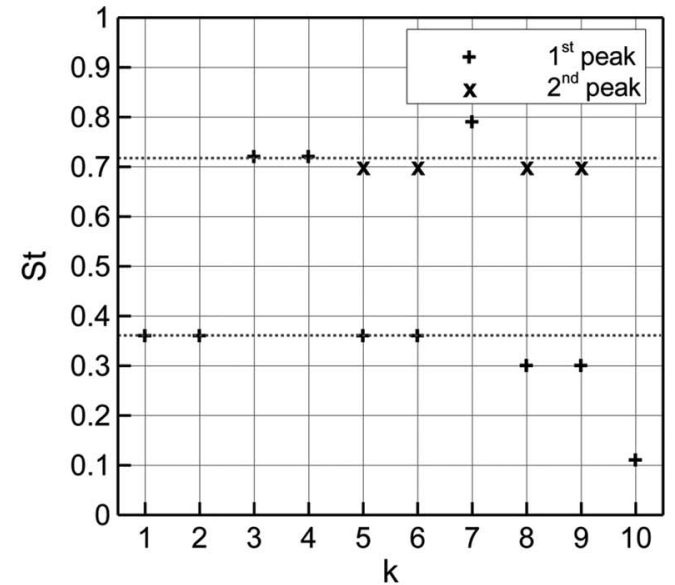

(b)

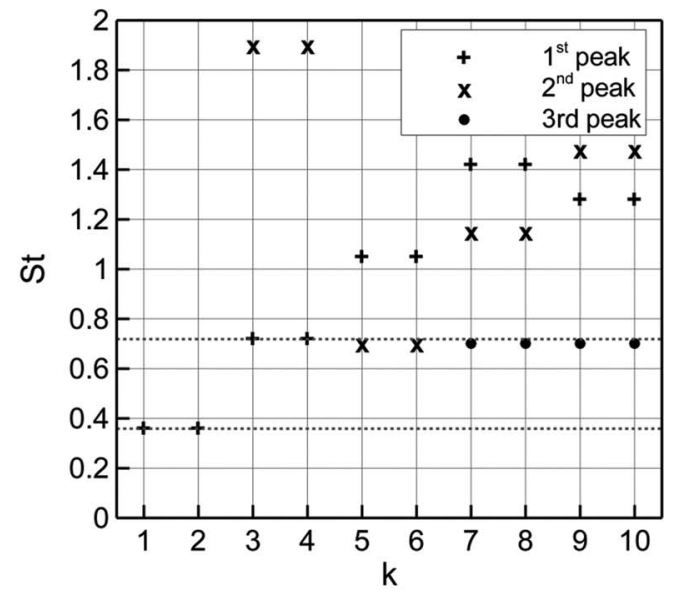

(d)

FIG. 23. Spectrum peaks of POD mode time coefficients. (a) $\mathbf{V}$ modes. (b) $\boldsymbol{\omega}$ modes. (c) $\mathbf{L}$ modes. (d) $d^{2} \mathbf{L} / d t^{2}$ modes. 
Patterns similar to modes 3-4 are observed for mode pairs 5-6, 7-8 and 9-10 also in the region across the end of the potential core $(5<Z / D<7)$. For conciseness, Figure 21 does not show the latter pair.

While velocity, vorticity, and Lamb vector modes are generally associated with values of the Strouhal number below 1, the modes of $d^{2} \mathbf{L} / d t^{2}$ are also associated to higher values (Figure 23), such as mode pair 3-4 $(S t=1.9)$, mode pair 5-6 $(S t=1.05)$, mode pair 7-8 $(S t=1.15$ and 1.42$)$ and mode pair 9-10 ( $S t=1.28$ and 1.48$)$.

\section{E. Noise prediction based on time-resolved tomographic PIV measurements}

The phenomenological analysis given so far shows that the experiments are conducted at a resolution that enables to appreciate the details of the vortex interactions and their connection to the local pattern of the Lamb vector and its second temporal derivative. In some recent studies, PIV measurements have been used in combination with aeroacoustic analogies to predict acoustic emissions. In the investigation of vortex-pairing sound in an acoustically-excited jet, Schram et al. ${ }^{25}$ applied phase-locked planar PIV to a conservative formulation of vortex sound theory for axisymmetric flow, observing good agreement between the acoustic prediction and microphone measurements. Following Schram et al., ${ }^{25}$ it was shown that, under the assumption of axisymmetric flow, time-resolved planar PIV can be applied to predict the sound of pairing vortices by direct evaluation of Powell's analogy (Violato et al.). With DNS tailored on the TR-TOMO PIV jet experiment of Violato and Scarano, ${ }^{19}$ Moore et al. ${ }^{51}$ showed that the acoustic source can be localized by a domain that extends from the nozzle exit to 3-4 jet diameters beyond the end of the potential core. In this chapter, we follow a methodology to predict the jet noise from the TR-TOMO PIV data using Powell's analogy.

When experimental data are concerned, the effective conservation of the flow impulse $\mathbf{I}$ and the kinetic energy $T$ can be used as a posteriori indicators of the suitability of the measurements for the sound prediction (Schram and Hirschberg ${ }^{52}$ ). Figure 24 shows the time history of axial and radial components of the flow impulse I and the kinetic energy $T$ (Eqs. (A2) and (A3) in the Appendix) evaluated over the measurement domain during 2 periods of vortex pairing. The trend shows negligible fluctuations ascribed to the flux through the outflow boundary. The normalized $\mathrm{rms}$ fluctuations $\left(r m s I_{r}=0.75 ; r m s I_{z}=0.53 ; r m s T=0.24\right)$ are smaller than $15 \%$ of the corresponding mean value and they are comparable with those reported by Schram. ${ }^{53}$

Acoustic predictions are performed based on Powell's analogy for a listener positioned at $90^{\circ}$ to the jet axis at a distance $R_{L}$. Thus, Eq. (A6) (see the Appendix) reduces to

$$
p^{\prime}(\mathbf{x}, t)=-\left.\frac{\rho_{0}}{4 \pi c_{0}^{2} R_{L}^{3}} \frac{\partial^{2}}{\partial t^{2}} \int_{Z_{1}}^{Z_{2}} \int_{0}^{2 \pi} \int_{0}^{R_{c}(Z)} y_{r} L_{r}\right|_{t^{*}} \operatorname{det}(\mathbf{J}) d R d \theta d Z,
$$

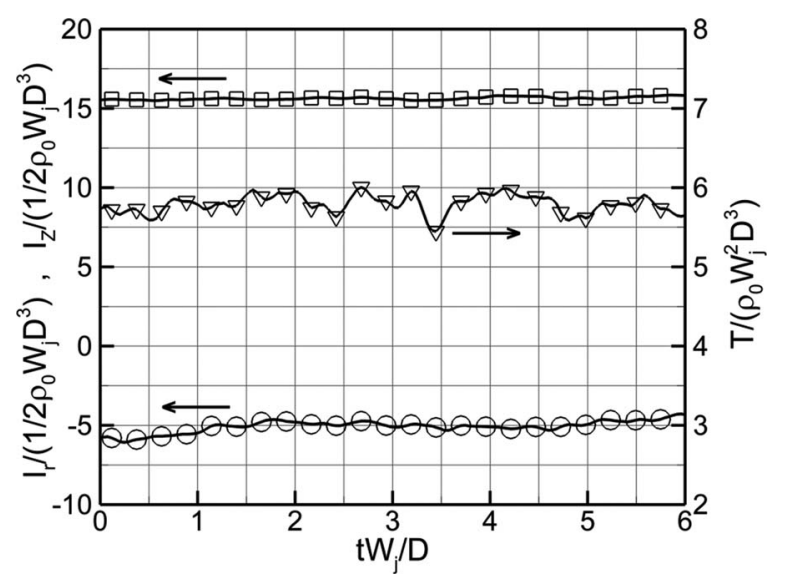

FIG. 24. Impulse and kinetic energy time history during 2 period of vortex pairing. Axial impulse $I_{z}$ (square symbol); radial symbol $I_{r}$ (circular symbol); kinetic energy $T$ (gradient symbol). 
where $R_{C}(Z)=0.065 Z+1$ is the radius function of the conical domain and $L_{r}$ is the radial component of the Lamb vector which is the only term accounting for the flow state.

The fluctuating component of $L_{r}, L_{r}^{\prime}=L_{r}-\overline{L_{r}}$ (where $\overline{L_{r}}$ is the mean value) is damped at the domain boundaries using flat-Hann window function following Obrist and Kleiser ${ }^{54}$ who showed its benefits in limiting the effects of source spatial truncation.

Considering damping lengths at the inflow, outflow, and radial boundary, respectively, $\alpha \Delta Z$, $\beta \Delta Z$ (where $\Delta Z=Z_{2}-Z_{1}$ ) and $\zeta R_{C}(Z)$ with $0 \leq \alpha \leq 1,0 \leq \beta \leq 1$ and $0 \leq \zeta \leq 1$, the flat-Hann window function along the axial direction reads as

$$
h_{Z}(Z)=\left\{\begin{array}{cc}
\frac{Z}{\alpha \Delta Z}-\frac{1}{2 \pi} \sin \left[\frac{2 \pi Z}{\alpha(\Delta Z)}\right] & Z_{1}<Z<\alpha \Delta Z \\
1 & \alpha \Delta Z \leq Z \leq(1-\beta) \Delta Z \\
\frac{(\Delta Z-Z)}{\beta \Delta Z}-\frac{1}{2 \pi} \sin \left[\frac{2 \pi(\Delta Z-Z)}{\beta \Delta Z}\right] & (1-\beta) \Delta Z \leq Z \leq Z_{2} \\
0 & \text { elsewhere }
\end{array},\right.
$$

while that along the radial direction is

$$
h_{r}(R)=\left\{\begin{array}{cc}
1 & 0 \leq R \leq \zeta R_{C}(Z) \\
\frac{\left(R_{C}(Z)-R\right)}{\zeta R_{C}(Z)}-\frac{1}{2 \pi} \sin \left[\frac{2 \pi\left(R_{C}(Z)-R\right)}{\zeta R_{C}(Z)}\right] & (1-\zeta) R_{C}(Z) \leq R \leq R_{C}(Z) \\
0 & \text { elsewhere }
\end{array}\right.
$$

Substituting Eqs. (16) and (17) into Eq. (15) gives

$$
p^{\prime}(\mathbf{x}, t)=-\frac{\rho_{0}}{4 \pi c_{0}^{2} R_{L}^{3}} \frac{\partial^{2}}{\partial t^{2}} \int_{Z_{1}}^{Z_{2}} \int_{0}^{2 \pi} \int_{0}^{R_{c}(Z)} y_{r}\left(\left.h_{z} h_{r} L_{r}^{\prime}\right|_{t^{*}}+\overline{L_{r}}\right) \operatorname{det}(\mathbf{J}) d R d \theta d Z .
$$

Acoustic predictions are performed for a listener located at a distance $R_{L}=10^{3} \mathrm{~m}$, which satisfies the far-field assumption $R_{L} \gg \lambda\left(\lambda=c_{0} / f\right.$, being $\lambda$ the typical acoustic wavelength, $c_{0}=1481 \mathrm{~m} / \mathrm{s}$ the speed of sound, and $f=15 \mathrm{~Hz}$ the pairing frequency; thus $\lambda=99 \mathrm{~m}$ ). The acoustic pressure spectrum illustrated in Figure 25 (black line) is obtained evaluating Eq. (18) with

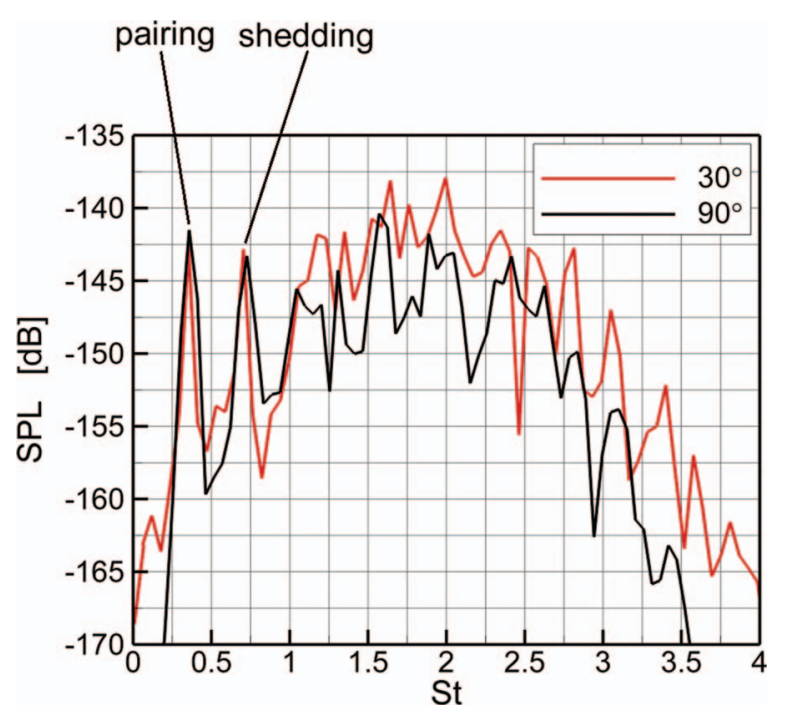

FIG. 25. Predicted acoustic spectrum. Listener at $30^{\circ}$ and $90^{\circ}$ to the jet axis; $R_{L}=10^{3} \mathrm{~m}$ (reference pressure: $20 \mu \mathrm{Pa}$ ). 


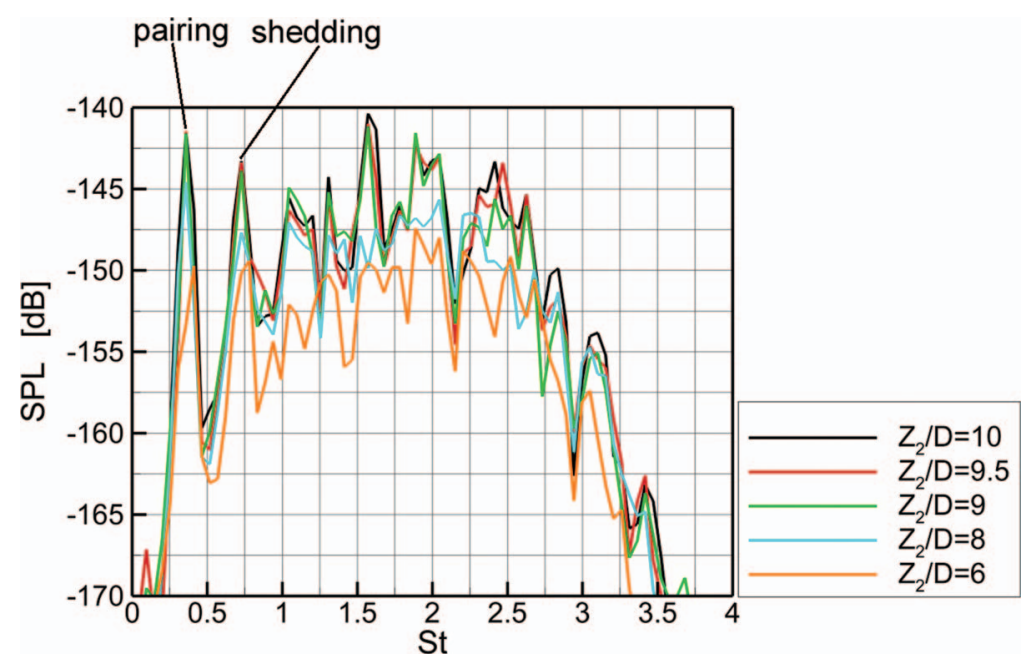

FIG. 26. Predicted acoustic spectra obtained for different axial lengths $Z_{2} / D$ of the domain (listener at $90^{\circ}$ to the jet axis; $R_{L}$ $=10^{3} \mathrm{~m}$; reference pressure $20 \mu \mathrm{Pa}$ ).

TR-TOMO PIV data between $Z_{1} / D=0$ and $Z_{2} / D=10$. It shows low-frequency peaks of sound pressure level $S P L=-141.5 \mathrm{~dB}$ and $-143.5 \mathrm{~dB}$ that, respectively, correspond to the vortex pairing $(S t=0.36)$ and shedding $(S t=0.72)$ frequencies, which is in agreement with Bridges and Hussain. ${ }^{55}$ It is remarked here that hydrophone measurements of such acoustic waves are unfeasible as they should be conducted at a distance of 1 kilometer from the jet.

The spectrum also shows smaller peaks at higher frequency, which belong to a broader hump $(1<S t<2.75)$. To understand the importance of spatial domain truncation on the acoustic spectra, acoustic predictions are conducted for axial lengths of the domain varying between 6 to 10 jet diameters and the obtained spectra are compared in Figure 26. When the domain extends from $Z_{I} / D$ $=0$ to $9 \leq Z_{2} / D \leq 10$, spectra show maximum variations of $2 \mathrm{~dB}$ for $S t \leq 2.25$. For shorter domains $\left(6 \leq Z_{2} / D \leq 8\right)$, in contrast, they exhibit larger amplitude drop and profile variations. From the above discussion, the estimated values of $S P L$ between $S t=0$ and 2.25 can be reasonably considered to be associated with flow events rather than domain spatial truncation. Note that the frequencies associated with three-dimensional waves of $d^{2} \mathbf{L} / d t^{2}$, evolving by the end of the potential core (Figure 23; modes 3-4: $S t=1.9$; modes 5-6: $S t=1.05$; mode 7-8: $S t=1.15$ and 1.42; modes 9-10: $S t=1.28$ and 1.48) where vortex rings disrupt (Figure 6) and the acoustic source is more pronounced (Figure 13), lie in the range of the acoustic spectral hump $(1<S t<2.25)$.

Exploiting the linearity of the integral function (see Eq. (15)), the acoustic source can be mapped by the second time derivative of the radial component of the Lamb vector $d^{2} L_{r} / d t^{2}$. Temporal sequences of such quantity during vortex ring breakdown show the existence of characteristic frequencies that are between 3 to 7 times higher than the pairing frequency. An example of "lifecycle" of the source is given in Figure 27, which shows the evolution of region " $\mathrm{A}$ " in a period of time $T W_{j} / D=0.45$, corresponding to $S t=2.1$, which agrees fairly well with the range of frequencies interested by the spectral hump ( $S t=1$ and 2.25) reported in Figure 25. This observation further confirms the dominant role of vortex ring disruption for acoustic production and collocates it in the frequency spectrum at values of the Strouhal more than two times larger than that of vortex shedding.

Finally, acoustic predictions are performed for a listener positioned at $30^{\circ}$ to the jet axis $\left(R_{L}=10^{3} \mathrm{~m}\right)$ using the data between $Z_{1} / D=0$ and $Z_{2} / D=10$. Similarly to Eq. (18), the flat-Hann damping functions $h_{z}$ (Eq. (16)) and $h_{r}$ (Eq. (17)) are substituted in Powell analogy (Eq. (A6)) where they are applied to the fluctuating components of the Lamb vector $\mathbf{L}^{\prime}=\mathbf{L}-\overline{\mathbf{L}}$ (where $\overline{\mathbf{L}}$ is the mean value). The equation that is then evaluated is

$$
p^{\prime}(\mathbf{x}, t)=-\frac{\rho_{0}}{4 \pi c_{0}^{2} R_{L}{ }^{3}} \frac{\partial^{2}}{\partial t^{2}} \int_{Z_{1}}^{Z_{2}} \int_{0}^{2 \pi} \int_{0}^{R_{c}(Z)}(\mathbf{x} \cdot \mathbf{y})\left[\mathbf{x} \cdot\left(\left.h_{r} h_{z} \mathbf{L}^{\prime}\right|_{t^{*}}+\overline{\mathbf{L}}\right)\right] \operatorname{det}(\mathbf{J}) d R d \theta d Z .
$$



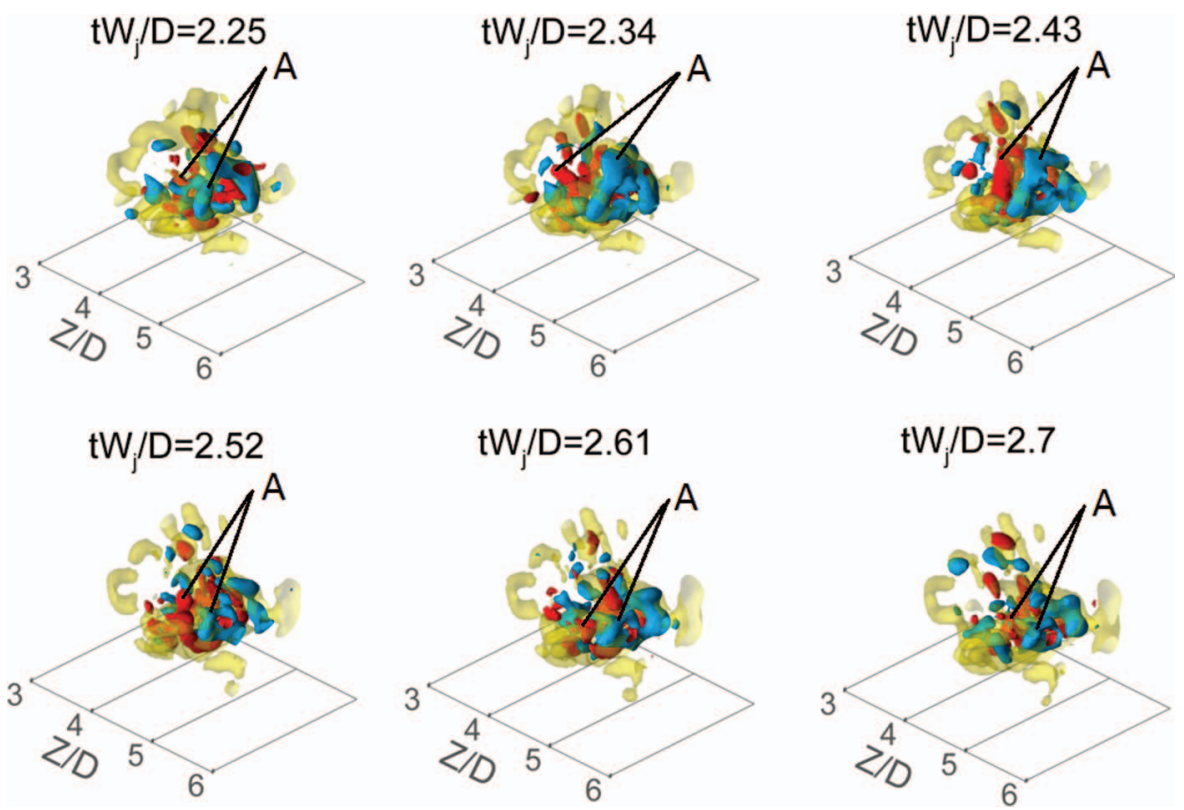

FIG. 27. Time sequence visualization of acoustic source during vortex ring breakdown. Iso-surfaces $\left(d^{2} L_{r} / d t^{2}\right) D^{3} / W_{j}^{4}$ $=-20$ (blue) and 20 (red); iso-surfaces $\lambda_{2} D / W_{j}=-0.8$ (yellow).

The resulting acoustic pressure spectrum (Figure 25, red line) shows peaks $S P L=-143 \mathrm{~dB}$ and $-141 \mathrm{~dB}$ that, respectively, correspond to the vortex pairing and shedding frequencies. This resembles the spectrum obtained at $90^{\circ}$, despite discrepancies of about $1 \mathrm{~dB}$. At $30^{\circ}$, the acoustic spectrum is characterized by a broader hump (only the range $1 \leq S t \leq 2.25$ is considered to be associated with flow events) featuring SPL levels typically $3 \mathrm{~dB}$ higher than those found at $90^{\circ}$. This result is in agreement the jet noise directivity previously documented (Lush; ${ }^{56}$ Stromberg et al. and $^{57}$ Bogey et al. ${ }^{4}$ )

\section{CONCLUSIONS}

The three-dimensional behavior of core breakdown in a transitional jet at $R e=5000$ is investigated by experiments conducted in a tailored-water jet facility with TR-TOMO PIV. The measurement domain features a truncated cone encompassing 10 jet diameters. This enables instantaneous measurements between the nozzle exit to about 4 jet diameters beyond the end of the potential core.

The flow exhibits a pulsatile motion according to the shedding and pairing of vortex rings, followed by the formation of counter-rotating pairs of streamwise vortices and the growth of four in-plane and four out-of-plane azimuthal instabilities. With the growth of in-plane azimuthal instabilities, vortex ring humps are tilted and ejected along the axial direction as they are subjected to higher axial velocities. By the end of the potential core, this process culminates in the breakdown of the toroidal shape into streamwise filaments oriented at $30^{\circ}-45^{\circ}$ to the jet axis and peripheral structures of " $\mathrm{C}$ " shape. The latter re-organize as filaments oriented along the azimuthal direction in the region downstream of the potential core and, are largely responsible for the flow entrainment. In the vicinity of the jet axis, instead, vortex filaments show a scattered orientation.

Peak activity of stretching and tilting fields is observed during the formation of "C" structures and the ejection of vortex humps, whereas it drops of $60 \%$ beyond the end of the potential core.

The relation between flow structures developing in the region of the jet core collapse and the instantaneous acoustic production is investigated recalling Powell's analogy. The second time derivative of the Lamb vector is associated with the acoustic source. Its spatio-temporal evolution is visually compared to that of the vortices, which are detected by $\lambda_{2}$-criterion, to identify flow events involved in the acoustic generation. The most intense source activity is observed by the end of the 
potential core when in-plane vortex ring humps are tilted and ejected leading to the ring breakdown. During this process, the source is observed to lose the initial toroidal shape and to congregate in the proximity of the jet axis.

The large scale flow organization is statistically investigated applying the snapshot-POD technique to the velocity and vorticity fields. While the velocity decomposition is based on the kinetic energy, the vorticity one is referred to the flow enstrophy. The attention is focused on the description of the first 10 modes, which capture $27.5 \%$ of the total kinetic energy in the velocity decomposition and $20 \%$ of the total flow enstrophy in the vorticity decomposition. The first pair of velocity and vorticity modes, which are the most energetic ones, describe the presence of vortex rings travelling after pairing. In the velocity modes, the axial velocity component identifies a helical motion in the region across the end of the potential core and, further downstream, a flapping motion. It also shows a precession motion that develops downstream the end of the potential core. On the other hand, the radial and the azimuthal components, respectively, identify wave-like and twisting motions between $Z / D=6$ and 10. In the vorticity decomposition, the first pair of modes shows that travelling vortex rings are characterized by the radial and axial vorticity fields developing across the end of the potential core with a characteristic $40^{\circ}-45^{\circ}$ inclination to the jet axis. Such patterns, also observed in mode pairs at lower energy, are ascribable to the process of vortex ring breakdown.

Snaphot-POD analysis is also applied to the Lamb vector and the second time derivative of the Lamb vector based on the $L_{2}$-norm of the fluctuating component. In Powell's aeroacoustic analogy, the former accounts for the flow state while the latter identifies the acoustic source. The attention is focused on the description of first 10 modes, which capture $30.3 \%$ of the total Lamb energy content in the Lamb vector decomposition and $31.9 \%$ of the total acoustic source energy in the Lamb vector second time derivative decomposition.

The first pair of Lamb vector modes describe vortex ring travelling after pairing, where the characteristic azimuthal coherence of the rings is identified by the radial and the axial components of the Lamb vector and not by the azimuthal component as seen in the corresponding vorticity modes. In the region across the end of the potential core, the motion of the rings is accompanied by the development of a three-dimensional disturbance at $40^{\circ}-45^{\circ}$ to the jet axis that is described by the azimuthal component. Travelling rings after shedding are identified by mode pairs at lower energy $3-4,5-6$, and 7-8. In these last two, for $Z / D>5$, the azimuthal component describes a travelling wave with axial swirling at an angle of $40^{\circ}-45^{\circ}$ to the jet axis.

In the Lamb vector second time derivative modes, the radial and axial components resemble a wave-packet organization with an axisymmetric pattern that gradually decays by the end of the potential core. This pattern is particularly clear in the first mode pair, where the radial and the axial components identify a travelling wave related to vortex pairing, but with halved spatial wavelength, featuring the growth of four primary azimuthal waves. The other four mode pairs are instead associated with the vortex shedding although they are characterized by halved spatial wavelength. In the region by the end of the potential core, where vortex rings disrupt and the acoustic source is more pronounced, the azimuthal component of modes 3-4 also describes the development of a three-dimensional disturbance at $40^{\circ}-45^{\circ}$ to the jet axis. Similar disturbances are detected by modes 5-6, 7-8 and 9-10 in the region across the end of the potential core.

Compared to velocity, vorticity, and Lamb vector modes, which are typically associated with Strouhal numbers smaller than 0.9, the mode 3-4, 5-6, 7-8, and 9-10 of $d^{2} \mathbf{L} / d t^{2}$ are also related to higher values $(1.05 \leq S t \leq 1.9)$, which are attributed to the three-dimensional disturbances developing in the region across the end of the potential core.

Far-field acoustic predictions are performed by direct evaluation of Powell's analogy with TRTOMO PIV data. For a far-field listener positioned at $90^{\circ}$ to the jet axis, the predicted acoustic spectrum shows peaks at the pairing and the shedding frequencies and several peaks belonging to a single broader hump in the frequency range $1 \leq S t \leq 2.75$. Frequencies $S t \leq 2.25$ are found to be unaffected by domain spatial truncation when the domain length is chosen to be greater than 9 diameters.

The frequencies that are related to the three-dimensional waves of the Lamb vector second time derivative modes ( $S t=1.9$, modes 3-4; $S t=1.05$, mode 5-6; $S t=1.15$ and 1.42, mode 7-8; 
$S t=1.28$ and 1.48, modes 9-10) lie in the range of the acoustic spectral hump $(1 \leq S t \leq 2.25)$, which, by the visual inspection of the acoustic source, can be ascribed to the breakdown of ring vortices.

Finally, far-field acoustic predictions are performed for a far-field listener at $30^{\circ}$ to the jet axis. The related spectrum shows peaks at pairing and shedding frequencies and a broader hump that, compared with the $90^{\circ}$ spectrum, lies in the same frequency range and shows SPL levels that are typically $3 \mathrm{~dB}$ higher.

\section{ACKNOWLEDGMENTS}

This work was conducted as part of the FLOVIST project (Flow Visualization Inspired Aeroacoustics with Time Resolved Tomographic Particle Image Velocimetry), funded by the European Research Council (ERC) (Grant No. 202887). The authors kindly acknowledge LaVision GmbH and Professor Piero Colonna for providing the CMOS cameras used in the TOMO- PIV experiments and Dr. Andrea Ianiro for contributing to the realization of the experiments.

\section{APPENDIX: FORMULATION OF POWELL'S ANALOGY IN CYLINDRICAL COORDINATES}

For low Mach numbers and compact source region, with no external force field and neglecting viscous-thermal effects, the far-field solution of Powell's analogy ${ }^{27}$ reads as

$$
p^{\prime}(\mathbf{x}, t)=-\frac{\rho_{0}}{4 \pi c_{0}^{2}|\mathbf{x}|^{3}} \frac{\partial^{2}}{\partial t^{2}} \iiint_{V}(\mathbf{x} \cdot \mathbf{y}) \mathbf{x} \cdot \mathbf{L} \mid d_{t^{*}} d^{3} \mathbf{y},
$$

where $\mathbf{x}$ is the listener's position vector, $\mathbf{y}$ is the source position vector, $\mathbf{L}$ is the Lamb vector evaluated at the retarded time $t^{*}=t-\frac{|\mathbf{x}|}{c_{0}}$ and $V$ is the three-dimensional domain. Exploiting the linearity of the integral function (see Eq. (A1)), the acoustic source can be mapped by the second time derivative of the Lamb vector $d^{2} \mathbf{L} / d t^{2}$.

Note that Equation (A1) is derived imposing the conservation of the flow impulse

$$
\mathbf{I}=\frac{1}{2} \rho_{0} \iiint_{V} \mathbf{y} \times \boldsymbol{\omega} d^{3} \mathbf{y}
$$

and the kinetic energy

$$
T=\rho_{0} \iiint_{V} \mathbf{y} \cdot(\boldsymbol{\omega} \times \mathbf{v}) d^{3} \mathbf{y}
$$

It is now considered a system of cylindrical coordinates $(R, \theta, Z)$ where $R, \theta$ and $Z$ are, respectively, the radial, the azimuthal and the axial coordinate. The listener's and the source position vectors are then written as

$$
\begin{aligned}
& \mathbf{y}=\left\{y_{r}, y_{\theta}, y_{z}\right\} \\
& \mathbf{x}=\left\{x_{r}, x_{\theta}, x_{z}\right\}
\end{aligned}
$$

while the Lamb vector reads as

$$
\mathbf{L}=\left\{L_{r}, L_{\theta}, L_{z}\right\}
$$

By substituting (A4) and (A5) into (A1), it is obtained the expression of Powell's analogy in cylindrical coordinates

$p^{\prime}(\mathbf{x}, t)=-\frac{\rho_{0}}{4 \pi c_{0}^{2}|\mathbf{x}|^{3}} \frac{\partial^{2}}{\partial t^{2}} \int_{Z_{1}}^{Z_{2}} \int_{0}^{2 \pi} \int_{0}^{R_{1}}\left(\left\{x_{r}, x_{\theta}, x_{z}\right\}\left\{\begin{array}{l}y_{r} \\ y_{\theta} \\ y_{z}\end{array}\right\}\right)\left(\left.\left\{x_{r}, x_{\theta}, x_{z}\right\}\left\{\begin{array}{l}L_{r} \\ L_{\theta} \\ L_{z}\end{array}\right\}\right|_{t^{*}}\right) \operatorname{det}(\mathbf{J}) d R d \theta d Z$, 
where $Z_{1}$ and $Z_{2}$ are the axial positions of the inflow and outflow boundaries, $R_{1}$ is the location of the radial boundary and

$$
\mathbf{J}=\left[\begin{array}{lll}
\frac{d x}{d R} & \frac{d x}{d \theta} & \frac{d x}{d Z} \\
\frac{d y}{d R} & \frac{d y}{d \theta} & \frac{d y}{d Z} \\
\frac{d z}{d R} & \frac{d z}{d \theta} & \frac{d z}{d Z}
\end{array}\right]=\left[\begin{array}{ccc}
\cos \theta-R \sin \theta & 0 \\
\sin \theta & R \cos \theta & 0 \\
0 & 0 & 1
\end{array}\right]
$$

is the Jacobian matrix.

${ }^{1}$ D. Juvé, M. Sunyach, and G. Comte-Bellot, "Intermittency of the noise emission in subsonic cold jets," J. Sound Vib. 71(3), 319-332 (1980).

${ }^{2}$ F. Hussain and K. B. M. Q. Zaman, "The preferred mode of the axisymmetric jet," J. Fluid Mech. 110, 39-71 (1981).

${ }^{3}$ F. Hussain, "Coherent structures and turbulence," J. Fluid Mech. 173, 303-356 (1986).

${ }^{4}$ C. Bogey, C. Bailly, and D. Juvé, "Noise investigation of a high subsonic, moderate Reynolds number jet using a compressible LES," Theor. Comput. Fluid Dyn. 16(4), 273-297 (2003).

${ }^{5}$ P. Jordan, M. Schlegel, B. R. Noack, and C. E. Tinney, "Identifying noisy and quiet modes in a jet," AIAA Paper 2007-3602.

${ }^{6}$ A. J. Yule, "Large structure in the mixing layer of a round jet," J. Fluid Mech. 89, 413-432 (1978).

${ }^{7}$ D. Liepmann and M. Gharib, "The role of streamwise vorticity in the near field entrainment of round jets," J. Fluid Mech. 245, 643-668 (1992).

${ }^{8}$ D. M. Kyle and K. R. Shreenivasan, "The instability and breakdown of a round variable-density jet," J. Fluid Mech. 249, 619-664 (1993).

${ }^{9}$ T. Matsuda and J. Sakakibara, "On the vortical structure in a round jet," Phys. Fluids 17, 025106 (2005).

${ }^{10}$ J. H. Cintriniti and W. K. George, "Reconstruction of the global velocity field in the axisymmetric mixing layer utilizing the proper orthogonal decomposition," J. Fluid Mech. 418, 137-166 (2000).

${ }^{11}$ D. Jung, S. Gamard, and W. K. George, "Downstream evolution of the most energetic modes in a turbulent axisymmetric jet at high Reynolds number. Part 1. The near-field region," J. Fluid Mech. 514, 173-204 (2004).

${ }^{12} \mathrm{M}$. O. Iqbal and F. O. Thomas, "Coherent structure in a turbulent jet via a vector implementation of the proper orthogonal decomposition,” J. Fluid Mech. 571, 281-326 (2007).

${ }^{13}$ K. P. Lynch and B. S. Thurow, "POD analysis of 3d-flow visualization images of a circular jet with Reynolds number 9500," AIAA Paper 2009-4303.

${ }^{14}$ B. Thurow and A. Satija, (2007). "Further Development of a high-speed three-dimensional flow visualization system," AIAA Paper No. 2007-1060, 2007.

${ }^{15}$ M. P. Wernet, "Temporally resolved PIV for space-time correlations in both cold and hot jet flows," Meas. Sci. Technol. 18, 1387-1403 (2007).

${ }^{16} \mathrm{C}$. Bruecker and W. Althaus, "Study of vortex breakdown by particle tracking velocimetry (PTV). Part 3: time-dependent structure and development of breakdown-modes," Exp. Fluids 18-3, 174-186 (1995).

${ }^{17}$ T. Hori and J. Sakakibara, "High-speed scanning stereoscopic PIV for 3D vorticity measurement in liquids," Meas. Sci. Technol. 15, 1067-1078 (2004).

${ }^{18}$ G. E. Elsinga, F. Scarano, B. Wieneke, and B. W. van Oudheusden, “Tomographic particle image velocimetry,” Exp. Fluids 41, 933-947 (2006).

${ }^{19}$ D. Violato and F. Scarano, "Three-dimensional evolution of flow structures in transitional circular and chevron jets," Phys. Fluids 23, 124104-124129 (2011).

${ }^{20}$ P. E. Dimotakis, R. C. Miake-Lye, and D. A. Papantoiou, "Structure dynamics of round turbulent jets," Phys. Fluids 26, 3185-3192 (1983).

${ }^{21}$ C. O. Paschereit, D. Oster, T. A. Long, H. E. Fiedler, and I. Wygnaski, "Flow visualization of interactions among large coherent structures in an axisymmetric jet," Exp. Fluids 12, 189-199 (1992).

${ }^{22}$ B. Ganapathisubramani, K. E. Longmire, and I. Marusic, "Investigation of three dimensionality in the near field of a round jet using stereo PIV," J. Turbul. 3, N16 (2002).

${ }^{23}$ S. C. Morris, "Shear layer instabilities: Particle image velocimetry measurements and implications for acoustics," Annu. Rev. Fluid Mech. 43, 529-550 (2011).

${ }^{24}$ J. M. Seiner, L. Ukeiley, and M. K. Ponton, "Jet noise source measurements using PIV," 5th AIAA/CEAS Aeroacoustics Conference and Exhibit, Bellevue, WA, AIAA Paper 99-1869 (1999).

${ }^{25}$ K. Schram, S. Taubitz, J. Anthoine, and A. Hirschberg, "Theoretical/empirical prediction and measurement of the sound produced by vortex pairing in a low Mach number jet," J. Sound Vib. 281, 171-187 (2005).

${ }^{26}$ V. Fleury, C. Bailly, E. Jondeau, M. Michard, and D. Juvé, "Space-time correlation in two subsonic jets using dual particle image velocimetry," AIAA J. 46, 2498-2509 (2008).

${ }^{27}$ A. Powell, “Theory of vortex sound,” J. Acoust. Soc. Am. 36, 177-195 (1964).

${ }^{28}$ J. Jeong and F. Hussain, "On the identification of a vortex," J. Fluid Mech. 285, 69-94 (1995).

29 J. B. Freund, "Noise sources in a low-Reynolds-number turbulent jet at Mach 0.9," J. Fluid Mech. 438, 277-305 (2001).

${ }^{30}$ F. Scarano and C. Poelma, "Three-dimensional vorticity patterns of cylinder wakes," Exp. Fluids 47, 69-83 (2009).

${ }^{31}$ G. Berkooz, P. Holmes, and J. L. Lumley, "The proper orthogonal decomposition in the analysis of turbulent flows," Annu. Rev. Fluid Mech. 25, 539-575 (1993). 
${ }^{32}$ B. Patte-Rouland, G. Lalizel, J. Moreau, and E. Rouland, "Flow analysis of an annular jet by particle image velocimetry and proper orthogonal decomposition," Meas. Sci. Technol. 12, 1404-1412 (2001).

${ }^{33}$ L. F. G. Geers, M. J. Tummers, and K. Hanjalic, "Particle imaging velocimetry-based identification of coherent structures in normally impinging multiple jets," Phys. Fluids 17, 055105 (2005).

${ }^{34}$ K. E. Meyer, J. M. Pedersen, and O. Ozcan, "A turbulent jet in crossflow analysed with proper orthogonal decomposition," J. Fluid Mech. 583, 199-227 (2007).

${ }^{35} \mathrm{M}$. El Hassan and A. Meslem, "Time-resolved stereoscopic particle image velocimetry investigation of the entrainment in the near field of circular and daisy shaped orifice jets," Phys. Fluids 22, 035107 (2010).

${ }^{36} \mathrm{M}$. El Hassan, A. Meslem, and K. Abed-Meraim, "Experimental investigation of the flow in the near-field of a cross-shaped orifice jet," Phys. Fluids 23, 045101 (2011).

${ }^{37}$ J. P. Schmid, D. Violato, and F. Scarano, "Decomposition of time-resolved tomographic PIV," Exp. Fluids 52, 1567-1579 (2012).

${ }^{38}$ L. Sirovich, "Turbulence and the dynamics of coherent structures. Part 1: Coherent structures," Q. Appl. Math. XLV, 561-571 (1987).

${ }^{39}$ J. Kostas, J. Soria, and M. S. Chong, "A comparison between snapshot POD analysis of PIV velocity and vorticity data," Exp. Fluids 38, 146-160 (2005).

${ }^{40}$ G. E. Elsinga, J. Westerweel, F. Scarano, and M. Novara, "On the velocity of ghost particles and bias errors in TomographicPIV,” Exp. Fluids 50, 825-838 (2011).

${ }^{41}$ M. Novara and F. Scarano, "Performances of motion tracking enhanced Tomo-PIV on turbulent shear flows," Exp. Fluids 52, 1027-1041 (2012).

${ }^{42}$ G. T. Hermann and A. Lent, "Iterative reconstruction algorithms," Comput. Biol. 6, 273-294 (1976).

${ }^{43}$ B. Wieneke, "Volume self-calibration for 3D particle image velocimetry," Exp. Fluids 45, 549-556 (2008).

${ }^{44}$ F. Scarano, "Tomographic PIV: principle and practice," Meas. Sci. Technol. 24, 012001 (2013).

${ }^{45}$ S. Crow and F. Champagne, “Orderly structure in jet turbulence," J. Fluid Mech. 48, 547-591 (1971).

${ }^{46}$ P. Bradshaw, D. H. Ferriss, and R. F. Johnson, "Turbulence in the noise producing region of a circular jet," J. Fluid Mech. 19, 591-624 (1964).

${ }^{47}$ W. Bi, Y. Sugii, K. Okamoto, and H. Madarame, "Time-resolved proper orthogonal decomposition of the near-field flow of a round jet measured by dynamic particle image velocimetry,” Meas. Sci. Technol. 14, L1-L5 (2003).

${ }^{48}$ A. M. Shinneeb, R. Balachandar, and J. D. Bugg, "Analysis of coherent structures in the far-field region of an axisymmetric free jet identified using particle image velocimetry and proper orthogonal decomposition,” J. Fluids Eng. 130, 011202 (2008).

${ }^{49}$ A. Cavalieri, G. Daviller, P. Comte, P. Jordan, G. Tadmor, and Y. Gervais, "Using large eddy simulation to explore sound-source mechanisms in jets," J. Sound Vib. 330, 4098-4113 (2011).

${ }^{50}$ A. Cavalieri, P. Jordan, A. Agarwal, and Y. Gervais, "Jittering wave-packet models for subsonic jet noise," J. Sound Vib. 330, 4474-4492 (2011).

${ }^{51}$ P. Moore, D. Violato, K. Bryon, and F. Scarano, "On the suitability of direct application of acoustic theory to time-resolved tomographic PIV tested by DNS for low Mach number jet flows," in Proceedings of the 16th AIAA/CEAS Aeroacoustic Conference, Melbourne, (Australia), August 25-28, 2009.

${ }^{52}$ C. Schram and A. Hirschberg, "Application of vortex sound theory to vortex-pairing noise: Sensitivity to errors in flow data," J. Sound Vib. 266(5), 1079-1098 (2003).

${ }^{53}$ C. Schram, "Aeroacoustic of subsonic jets: Prediction of the sound produced by vortex pairing based on particle image velocimetry," Ph.D. dissertation, Eindhoven University, The Netherlands.

${ }^{54}$ D. Obrist and L. Kleiser, "The influence of spatial domain truncation on the prediction of acoustic far-fields," AIAA Paper 2007-3725.

${ }^{55}$ J. E. Bridges and A. K. M. F. Hussain, "Roles of initial condition and vortex pairing in jet noise," J. Sound Vib. 117, 289-311 (1987).

${ }^{56}$ P. A. Lush, "Measurements of subsonic jet noise and comparison with theory," J. Fluid Mech. 46(3), 477-500 (1971).

${ }^{57}$ J. L. Stromberg, D. K. McLaughlin, and T. R. Troutt, "Flow field and acoustic properties of a Mach number 0.9 jet at low Reynolds number," J. Sound Vib. 72, 159-176 (1980). 